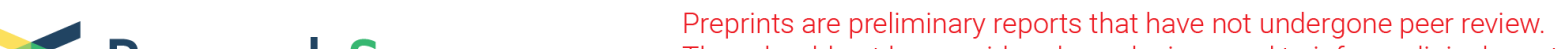 Research Square
or referenced by the media as validated information.
}

\section{Skeletal muscle fiber-type switching, myopathy, and exercise intolerance in Sigmar1 null mice}

\section{Richa Aishwarya}

Louisiana State University Health Sciences Center Shreveport

\section{Chowdhury Abdullah}

Louisiana State University Health Sciences Center-Shreveport https://orcid.org/0000-0001-5771-220X

\section{Naznin Remex}

Louisiana State University Health Sciences Center Shreveport

\section{Shafiul Alam}

Louisiana State University Health Sciences Center Shreveport https://orcid.org/0000-0001-8684-624X

\section{Mahboob Morshed}

Louisiana State University Health Sciences Center Shreveport

\section{Sadia Nitu}

Louisiana State University Health Sciences Center Shreveport

\section{Brandon Hartman}

Louisiana State University Health Sciences Center Shreveport

\section{Judy King}

Louisiana State University Health Sciences Center Shreveport

\section{Mohammad Alfred Nobel Bhuiyan}

Cincinnati Children's Hospital Medical Center

\section{A. Orr}

LSU Health Sciences Center - Shreveport

Christopher Kevil

LSU Health Shreveport https://orcid.org/0000-0003-0863-7260

\section{Md. Shenuarin Bhuiyan ( $\square$ mbhuiy@lsuhsc.edu )}

Louisiana State University Health Sciences Center Shreveport https://orcid.org/0000-0003-0073-3071

\section{Article}

Keywords: Sigmar1, Skeletal muscle, switching, myopathy, exercise intolerance

Posted Date: April 26th, 2021

DOI: https://doi.org/10.21203/rs.3.rs-399538/v1 
License: (c) (i) This work is licensed under a Creative Commons Attribution 4.0 International License. Read Full License 


\section{Abstract}

Sigmar1 is a widely expressed, multitasking molecular chaperone protein playing functional roles in several cellular processes. Mutations in the Sigmar1 gene have been reported to associate with several motor neuropathies, including amyotrophic lateral sclerosis, distal hereditary motor neuropathy, silver-like syndrome, and frontotemporal lobar degeneration. All these human mutations associated with motor neuropathies show strong manifestation in skeletal muscle with phenotypes like muscle wasting and atrophy. However, the physiological function of Sigmar1 in skeletal muscle remains unexplored. Here, we determined the physiological role of Sigmar1 in skeletal muscle structure and function in five different skeletal muscles: gastrocnemius (Gastro), quadriceps (Quad), soleus (Sol), extensor digitorum longus (EDL), and tibialis anterior (TA). Quantification of myofiber cross-sectional area (CSA) showed altered muscle mass and a slow-to-fast fiber-type switch in the skeletal muscle fibers of the Sigmar1 $1^{-/-}$mice. Interestingly, ultrastructural analysis by transmission electron microscopy showed the presence of tubular aggregates in the type I myofibers (Gastro, Quad, and TA) of Sigmar1 ${ }^{-/-}$mice. Immunostaining also showed derangements in dystrophin localization in skeletal muscles from Sigmar1 ${ }^{-/-}$mice. Additionally, skeletal muscle myopathy in Sigmar $1^{-/-}$mice was associated with an increased number of central nuclei, increased collagen deposition, and fibrosis. Functional studies also showed reduced endurance and exercise capacity in the Sigmar1 ${ }^{-/-}$mice. Overall, our studies demonstrated, for the first time, a potential physiological function of Sigmar1 in the skeletal muscle in maintaining healthy muscle structure and function.

\section{Introduction}

Sigmar1 is a multifunctional chaperone protein ubiquitously expressed in multiple organs [1-6]. Extensive studies demonstrate several molecular functions of Sigmar1 in different cell types, including regulation of ion channel function, calcium signaling, mitochondrial function, autophagy, and lipid transport [7-10]. Since the discovery over 40 years ago [11] [12], several recessive mutations in Sigmar1 have been associated with amyotrophic lateral sclerosis (ALS), distal hereditary motor neuropathy (dHMN), frontotemporal lobe degeneration (FTLD), and silver-like syndrome.

Most of the genetic studies on Sigmar1 demonstrated an association with Sigmar1 mutations to ALS pathology. The clinical hallmarks of ALS pathology include progressive muscle wasting, speech and swallowing difficulties, fasciculation, altered reflexes, spasticity, and death due to respiratory complications[13]. Juvenile cases of ALS were associated to a missense mutation (c.304G > C, p.E102Q) [14] and a frameshift mutation (c.283dupC, p.L95 fs)[15] in Sigmar1. Progressive development of skeletal muscle pathology was observed in E102Q mutations bearing patients, including weakness of hand and forearm muscles (at the age of 9 to 10 years) leading to paralysis of forearm extensors and triceps. These patients did not show respiratory or bulbar muscle weakness and showed normal sphincteric, sensory, and cerebellar functions [14]. Similarly, the patient with the L95 fs mutations developed progressive muscle weakness with significant atrophy of distal muscles with development of pes cavus 
and wasting of calf muscles and intrinsic muscles of the hands [15]. Interestingly, examination of a biopsy of vastus lateralis muscle in showed severe type II fiber predominance with scattered angular esterase positive fibers and also showed intense staining with nicotinamide adenine dinucleotide tetrazolium reductase (NADH-TR) [15]. Patients bearing these mutations showed normal brain and spinal cord magnetic resonance imaging (MRI) [14, 15].

All these clinical skeletal muscle phenotypes observed in the Sigmar1 mutation-bearing patients also observed in patients with distal hereditary motor neuropathy (dHMN). In fact, several truncations/deletions or point mutations in Sigmar1 were also reported in association with the development of dHMN [16-21]. The dHMN comprise a heterogeneous group of diseases having the common feature of slowly progressive, symmetrical, and distal-predominant neurogenic weakness and amyotrophy. All the dHMN patients with Sigmar1 mutation manifest identical clinical features of progressive muscle wasting/weakness in lower and upper limbs without sensory loss [16-21] with normal brain and spine MRI[17].

Studies also showed the association of Sigmar1 mutations in the 3'-untranslated region with the frontotemporal lobar degeneration (FTLD)-motor neuron disease (MND) pathological ubiquitinated inclusion bodies observed. Sigmar1 normally localizes to cytoplasmic membranes in healthy individuals, while in the c. $672 * 51 \mathrm{G}>T$ carriers showed intense Sigmar1 immunoreactivity in the nucleus dentate granule and CA1 pyramidal cells. However, the details about the clinical features of these patients remain unknown. Patients bearing a homozygous missense variant (c.194T > A, p.Leu65GIn) in Sigmar1 were associated with autosomal recessive Silver-like syndrome[18]. The clinical feature of this Sigmar1 mutation-bearing patient includes bilateral foot drop and frequent falls (at age 3 years), with the developed progressive muscle weakness and atrophy in the lower limbs. This patient developed clawed hands with no fixed contractures, bilateral finger and foot drop, knee bobbing, marked muscle atrophy from mid-forearms and knees down, and weakness of wrist extension at the age of 17 years. However, this patient has normal intellect, no sensory symptoms, and no sphincter problems with normal brain and spinal cord MRIs.

Despite all these reports, a direct association of mutations in Sigmar1 with human diseases remains elusive as identified only in small and isolated families, with limited genetic and functional studies. Functional studies to determine molecular mechanism showed that ALS associated Sigmar1 mutations (p.E102Q and p.L95 fs) $[14,15]$ are uniformly unstable and non-functional when expressed in Neuro2a (N2a) cells, suggesting a role of Sigmar1's loss of function in ALS [14, 15]. Moreover, expression of the Sigmar $1^{\mathrm{E} 102 \mathrm{Q}}$ carrying mutation in Drosophila (which lacks a Sigmar1 homolog) alters locomotor activity and eye development[22]. Whereas functional studies using two of dHMN associated mutations (p.E138Q and p.E150K) in several neuronal cell lines (two human neuroblastoma cell lines, SH-SY5Y and SK-N-BE, and the murine motor neuron-like NSC-34 line) suggested the pathogenicity of the mutations may involve the alterations in ER-mitochondria tethering, calcium homeostasis, and autophagy. The presence of the c. $672 * 26 \mathrm{C}>\mathrm{T}, \mathrm{c} .672 * 47 \mathrm{G}>\mathrm{A}$, and c. $672 * 51 \mathrm{G}>\mathrm{T}$ mutations within the $3{ }^{\prime}-\mathrm{UTR}$ of SIGMAR1 affect transcript stability resulting in increased Sigmar1 transcript in human neuroblastoma SK-N-MC 
and HEK293 cells [23]. Though studies using Sigmar1 global knockout mice (Sigmar $1^{-/-}$) provided a molecular tool to understand the physiological function of Sigmar1 [24, 25], these mice did not show any pathological phenotype associated with the human diseases observed in Sigmar1 mutation bearing patients. The neuronal dysfunction reported in Sigmar $1^{-/-}$mice were locomotor defects [26], nerve denervation [25], loss of motor neurons [25], age-dependent motor dysfunction [27], and development of depressive-like behavior $[24,28]$.

The most common clinical feature observed in Sigmar1 mutation-bearing patients is muscle weakness that may be caused by the upper or lower motor neuron injury resulting in denervation, or it may be the result of myofiber injury, resulting in a primary myopathy process. However, the physiological function of Sigmar1 in the skeletal muscle had never been studied and remains elusive. In this present study, we determined the physiological role of Sigmar1 in skeletal muscle physiology and function using the Sigmar1 null mice. We extensively evaluated the functional consequences of Sigmar1 ablation in skeletal muscle morphology, histology, ultrastructure, and function in different muscle fibers using the Sigmar ${ }^{-/-}$ mice. Here, we reported altered myofiber cross-sectional area, fibrotic remodeling, accumulation of tubular aggregates, abnormal mitochondria, and reduced endurance and exercise capacity in Sigmar1 ${ }^{-/-}$mice compared to the wildtype littermate control mice.

\section{Results}

\section{Sigmar1 expression and localization in myofibers}

Sigmar1 is a widely expressed molecular protein in mammalian cell systems. Herein, we first confirmed Sigmar1's expression in five different skeletal muscles, including Gastrocnemius (Gastro), Quadriceps (Quad), Tibialis Anterior (TA), Soleus (Sol), and Extensor Digitorum Longus (EDL) muscles isolated from wildtype (Wt) mice. Sigmar1 protein level quantified by Western blot analysis (Fig. 1a-b) and mRNA expression levels (Fig. 1c) by qPCR showing the differential expression of Sigmar1 in all these five muscles. Next, we visualized Sigmar1's localization and expression pattern in myofibers by immunofluorescence staining using anti-Sigmar1 antibody targeting the N-terminal (Fig. 1d) and Cterminal (Fig. 1e) of Sigmar1 on Quad muscle sections. We used sarcomere-associated anti-a-sarcomeric actinin co-immunostaining as a myofibers marker. Anti-Sigmar1 staining reveals differential expression of Sigmar1 across the myofibers. We observed comparatively intense anti-Sigmar1 fluorescence staining (in green) in myofibers with smaller diameters than larger diameter myofibers. Anatomically, slow-twitch, oxidative myofibers (type I) have smaller diameters than fast-twitch, glycolytic myofibers (type II) $[29,30]$. To confirm whether Sigmar1 is highly expressed in oxidative type I myofibers, we stained Quad myofibers with N-terminal targeted anti-Sigmar1 antibody with counterstaining with an anti-Type I myosin (Fig. 1f) and anti-OXPHOS (Fig. 1g) antibodies, respectively. Coherent with anatomical distinctions, anti-Sigmar1 immunostaining appeared at higher fluorescence intensity (in green) in anti-Type I myosin (in red) stained small, oxidative type I myofibers (Fig. 1f). We further confirmed these findings as anti-OXPHOS stained (in red) mitochondria rich, oxidative, smaller diameter type I myofibers contained high Sigmar1 expression (anti-Sigmar1 stained immunofluorescence in green) (Fig. 1g). Here, we confirmed the expression of 
Sigmar1 across different types of skeletal muscles and observed the higher expression of Sigmar1 in mitochondria-rich, oxidative, smaller diameter type I myofibers.

\section{Sigmar $1^{-/-}$mice myofibers exhibit altered myofiber areas in skeletal muscles}

Mammalian skeletal muscle composed of remarkably heterogeneous myofibers in terms of major contractile motor units, metabolic enzyme activity, and mitochondrial abundance to support a wide variety of physical demands through generating force and movement to accomplish maintenance of posture, respiration, locomotion, and intense activities. To investigate whether Sigmar1 plays any physiologically essential functions in maintaining normal muscle morphology, histology, and functions in adulthood, we have utilized genetic Sigmar1 knockout $\left(\right.$ Sigmar $1^{--}$) mice with respective age-matched littermate Wt mice as controls. Next, we assessed gross and microscopic morphometry of Gastro, Quad, TA, Sol, and EDL muscles isolated from Wt and Sigmar1 $1^{--}$mice (Fig. 2a-e). Morphologically all these isolated muscles showed no visible difference and had similar weights. Measurements of muscle weightto-tibia length ratio by gravimetric studies in freshly isolated skeletal muscles from Wt and Sigmar ${ }^{-/}$ mice showed similar muscle mass (Fig. 2a-e). We have confirmed the ablation of Sigmar1 protein in respective skeletal muscles compared to littermate Wt mice (Fig. 2f).

As the muscle showed normal morphometry, we measured myofibers cross-sectional areas (CSA) using wheat germ agglutinin (WGA) staining of the histological sections of skeletal muscles isolated from Wt and Sigmar1 ${ }^{\%-}$ mice (Fig. 3a-t). Interestingly, the quantified myofibers CSA in Sigmar ${ }^{\%-}$ mice showed differential changes depending on the anatomically distinct muscle types compared to Wt mice. Gastro (Fig. 3a-c) and Quad (Fig. 3e-g) myofibers in Sigmar1/- mice showed a significant increase in myofibers CSA with a clear shift towards larger size as shown by their respective size distribution in muscle sections. While TA (Fig. 3i-k), Sol (Fig. 3m-o), and EDL (Fig. 3q-s) myofibers showed a significant decrease in mean myofiber CSA with a shift towards smaller size as shown by their respective size distribution in muscle sections from Sigmar $1^{-/}$mice compared to Wt mice. Central nuclei in skeletal muscles are associated with different myopathies, developmental abnormalities, or muscle regeneration [31-34]. Sigmar1 ${ }^{-/-}$mice showed increased myofiber central nuclei in Gastro (Fig. 3d), Quad (Fig. 3h), and TA (Fig. 3l) muscles compared to the Wt mice. However, the central nuclei number did not change significantly across groups in the Sol (Fig. 3p) and EDL (Fig. 3t) (though it showed an increasing pattern in EDL). Overall, the Sigmar1 ${ }^{--}$mice showed hypertrophied myofibers in Gastro and Quad muscle sections and an increased number of myofiber central nuclei in Gastro, Quad, and TA muscle sections compared to Wt mice muscles.

\section{Sigmar $1^{-/-}$mice myofibers showed the myofiber-type switch}

Under different pathological conditions, myofibers types switch from slow-to-fast or fast-to-slow fiber types $[35,36]$. As Sigmar1 showed higher expression in mitochondria-rich type I myofibers, we immunostained histological sections of the Gastro, Quad, TA, Sol, and EDL muscles isolated from Wt and Sigmar $1^{--}$mice with anti-Myosin Type I antibody (red) and WGA (green) (Fig. 4a, f, k, p, u). Quantification 
of the myofibers showed a significant reduction in type I myofibers in contrast with a substantial increase in type II myofibers per microscopic field in Gastro and Quad muscles of Sigmar1 ${ }^{-/-}$mice suggesting a switch in muscle fiber types from slow-to-fast (i.e., from Type I to type II) (Fig. $\mathbf{4 b}, \mathbf{c}, \mathbf{g}, \mathbf{h}$ ). There were no significant changes in the percent number of type I and type II fibers in TA, Sol, and EDL muscles between genotypes (Fig. $\mathbf{4}, \mathbf{m}, \mathbf{q}, \mathbf{r}, \mathbf{v}, \mathbf{w}$ ). Along with these changes, Gastro and Quad muscle fibers of Sigmar $1^{-1}$ mice showed significantly increased cross-sectional areas of both type I and type II fibers compared to Wt mice (Fig. 4d, e, i, j). In contrast, both type I and type II myofiber areas were significantly decreased in TA and EDL muscles of Sigmar1 ${ }^{-/}$mice compared to Wt mice (Fig. $4 \mathbf{n}, \mathbf{0}, \mathbf{x}, \mathbf{y}$ ). There were no significant changes in myofiber areas in soleus muscles between genotypes (Fig. 4s, $t$ ). Overall, we found an aberrant alteration in myofibers numbers with accompanying changes in myofibers size in Sigmar ${ }^{-1}$ mice skeletal muscles compared to littermate Wt mice.

\section{Sigmar $1^{-/-}$mice skeletal muscles exhibit tubular aggregates and aberrant dystrophin localization}

Mutations in the SIGMAR1 gene have been reported to be associated with several neuropathies with severe pathological manifestations in skeletal muscles [14-19, 23, 37-42]. Moreover, ultrastructural examination by transmission electron microscopy (TEM) also showed altered mitochondrial organization in motor neurons [25] and cardiac muscles. Therefore, we examined the ultrastructure of the Gastro, Quad, TA, Sol, and EDL muscles isolated form Wt and Sigmar1 ${ }^{-/-}$mice (Fig. 5a-e). TEM examination of Wt mice skeletal myofibers showed normal ultrastructure and sarcomere organization in mitochondria deficient type II myofibers (Fig. 5a-e). Interestingly, Sigmar ${ }^{-/-}$mice Gastro, Quad, and TA muscles exhibit abnormal accumulation of crystalline-like tubular aggregates between myofibrils and beneath the sarcolemma in type II muscle fibers (Fig. 5a-c). Tubular aggregates are the dense accumulation of sarcoplasmic reticulum tubules, which are the characteristic feature of a rare genetic condition called tubular aggregate myopathy $[43,44]$. Mitochondria-rich type I myofibers of Wt mice skeletal muscles exhibited normal mitochondria organization at subsarcolemmal and intermyofibrillar spaces (Fig. 5a-e). In contrast, Sigmar $1^{\%}$ mice muscles show abnormal, amorphous shaped mitochondria accumulation in type I myofibers (Fig. 5a-e).

Due to the presence of tubular aggregates, we also observed the immunostaining with dystrophin to determine the sarcolemma integrity. In healthy muscle cells, dystrophin is localized between the sarcolemma and the outermost layer of myofilaments in the myofibers. Immunostaining of dystrophin (red) with counterstaining with WGA (green) showed normal sarcolemmal organization in Gastro, Quad, TA, Sol, and EDL muscles isolated from Wt mice (Fig 6a-e). Strikingly, Gastro, Quad, and TA muscles of Sigmar $1^{--}$mice showed the dislocation of dystrophin from sarcolemma and aberrant anti-dystrophin stained aggregates in skeletal muscles (Fig 6a-c). Overall, Sigmar ${ }^{-/-}$mice skeletal muscles showed abnormal tubular aggregates, abnormal mitochondria accumulation, and aberrant dystrophin aggregates in the muscles.

\section{Increased collagen deposition and fibrosis in Sigmar $1^{-/-}$mice skeletal muscles}


Skeletal muscles from Sigmar ${ }^{1-}$ mice showed pathological features with myocytes hypertrophy, muscle fiber type switching, and the presence of tubular aggregates. We further evaluated fibrotic remodeling in the histological sections of skeletal muscles (Gastro, Quad, TA, Sol, and EDL muscles) isolated from Wt and Sigmar1 ${ }^{-1-}$ mice with Picro-Sirius red (PSR) (Fig. 7a-e) staining and Masson's Trichrome staining (Fig. 8a-e). All the five skeletal muscles of Sigmar $1^{-/-}$mice exhibited significantly increased collagen deposition in the interstitial and perivascular region compared to Wt mice muscle sections (Fig. 7a-e). Similarly, Masson's Trichrome staining showed increased fibrosis areas in all five skeletal muscles of Sigmar $1^{-/-}$mice compared to Wt mice muscles (Fig. 8a-e). Moreover, Sigmar $1^{\%-}$ mice muscles exhibited central nuclei (black arrows) in myofibers compared to peripheral nuclei in Wt mice muscle sections in H\&E staining (Fig. 9a-e), recapitulating our findings in fluorescence studies (Fig. 3). We also observed an increase in interstitial eosinophilic collagen deposition (bright pink fibers) in Sigmar $1^{-/}$ mice muscle sections compared to Wt mice muscle sections.

\section{Sigmar $1^{-/-}$mice exhibit reduced endurance and exercise capacity}

As the myofibers of Sigmar1 $1^{-/}$mice exhibited an array of histopathological phenotype, performed a functional assessment of the skeletal muscle using grip strength measurement and graded maximal exercise testing following acclimatization (Fig. 10a-f)). Grip strength measurements showed lower values for both absolute values (Fig. 10a) and normalized values (Fig. 10b) in the Sigmar $1^{-1-}$ mice compared to their littermate Wt control mice indicating reduced physical endurance in Sigmar $1^{-1-}$ mice. Similarly, forced treadmill running also showed that Sigmar ${ }^{-/-}$mice have a compromised ability to run on a treadmill indicated by reduced exhaustion time (Fig 10c), maximum distance run (Fig 10d), maximal speed (Fig 10e), and average speed (Fig 10f) compared to littermate Wt mice. Overall, Sigmar $1^{-/-}$mice showed reduced muscle strength corroborating with attenuation in endurance and tolerance to exercise.

\section{Discussion}

This study aimed to extensively characterize the skeletal muscle morphology, histology, ultrastructure, and function in Sigmar1 null mice to demonstrate the physiological function of Sigmar1 in the skeletal muscles (Gastro, Quad, TA, Sol, and EDL). We have several intriguing findings in the current study, as follows: (i) Sigmar1 is differentially expressed in skeletal muscles with higher expression in type I (oxidative) muscle fibres compared to type II (glycolytic) muscle fibres; (ii) Quantification of myofiber CSA showed altered muscle mass and a slow-to-fast fiber-type switch in the skeletal muscle fibers from the Sigmar1 ${ }^{-/-}$mice; (iii) Ultrastructural analysis by TEM showed the presence of tubular aggregates in the type I muscle fibers (Gastro, Quad and TA) and abnormal mitochondria accumulation in type II muscle fibers of Sigmar1 ${ }^{-/-}$mice; (iv) Immunostaining showed derangement of dystrophin localization in muscles (Gastro, Quad and TA) of Sigmar1 ${ }^{-/-}$mice; (v) Sigmar1 ${ }^{-/-}$mice showed an increased number of central nuclei (H\&E staining), increased collagen deposition (Picro-Sirius red staining), and fibrosis (Masson's trichrome staining); (vi) Finally, functional studies showed Sigmar1 ${ }^{-/-}$mice had reduced endurance and exercise capacity compared to Wt mice. Overall, our studies demonstrated, for the first 
time, a potential physiological function of Sigmar1 in the skeletal muscle in maintaining healthy muscle structure and function.

Extensive studies on Sigmar1 biology to date demonstrated an array of beneficial cellular functions in multiple organs, including the brain, heart, liver, and bladder. The most-reported neurological dysfunction in Sigmar1 null mice were locomotor defects [26], significant nerve denervation [25], loss of motor neurons [25], age-dependent motor phenotype [27], and showed a depressive-like behavior [24, 28]. Additionally, the lack of Sigmar1 in the liver showed increased oxidative and metabolic stress with increased anaerobic metabolism $[45,46]$. Extensive studies on cardiac muscles of Sigmar1 null mice showed mitochondrial dysfunction, abnormal mitochondrial architecture, adverse cardiac pathological remodeling, and development of cardiac contractile dysfunction [47]. In addition, Sigmar1 activation by treatment with ligands showed cardioprotective effects reducing cardiac hypertrophy in an animal model of cardiac injury [48-53]. Though there are similarities among the major intracellular components of both cardiac and skeletal muscle cells (i.e., mitochondria, sarcoplasmic reticulum, and the myofibrils), the skeletal muscle differs substantially in its structure, regulation, and function. In this study, we first time explored the effects of Sigmar1 ablation in muscle histochemical and ultrastructural alteration using the Sigmar1 null mice.

Skeletal muscles consist of a heterogeneous mixture of type I (oxidative) and type II (glycolytic) myofibers. The proportions of these myofibers in skeletal muscle vary depending on the nature, location, and function of skeletal muscle. For instance, the fast-twitch muscle like quadriceps muscles consists of a large proportion of type II myofibers, whereas the slow-twitch muscle like Sol comprises primarily of type I myofibers. Type I or oxidative myofibers are mitochondria-rich myofibers relying mostly on $\beta$ oxidation for energy production whereas, type II or glycolytic myofibers contains a higher dependency on glycolytic pathways for energy production [29,30]. We observed the Sigmar1 expression rich in type 1 mitochondria-rich myofibers, and Sigmar1 ${ }^{-/-}$myofibers showed a switch from low-to-fast fiber-type. The differential effect of Sigmar1 on altering myofiber CSA can be attributed to the skeletal muscle's mixed myofiber composition: glycolytic myofibers rich muscles exhibited either increase or decrease in myofiber CSA whereas mitochondria rich oxidative myofibers showed no change in the Sigmar1 null muscle.

All the reported Sigmar1 mutation-bearing patients develop manifestations in skeletal muscle. Among these Sigmar1 mutations, the patient with the $L 95 \mathrm{fs}$ develops muscle weakness with significant atrophy and wasting of calf muscles and intrinsic muscles of the hands [15]. This patient showed severe type II fiber predominance with scattered angular esterase positive fibers in a biopsy of vastus lateralis muscle [15], a type 1 myofiber rich quadriceps muscle in healthy humans [54]. Myofibers switch from type I to type II or type II to type I had also been reported under pathological conditions [35, 36]. Interestingly, the p.L95 fs Sigmar1 mutant was unstable and non-functional when expressed in Neuro2a (N2a) cells, suggesting a role of Sigmar1's loss of function in the development of pathology. Interestingly, we also observed a significantly increased type II myofibers in muscles (Quad) of the Sigmar1 null mice suggesting a role of Sigmar1 in maintaining myofiber types. The molecular mechanisms of differential 
changes in myofiber type switch across the muscles in Sigmar1 mice ${ }^{-/-}$remains unknown and requires further studies.

The most intriguing phenotype observed in the skeletal muscles (Gastro, Quad, and TA) from Sigmar1 ${ }^{-/-}$ mice were the accumulation of tubular aggregates in type II muscle fibers, abnormal mitochondria accumulation in type I muscle fibers, and derangement in dystrophin localization. Interestingly, the skeletal muscle pathology observed in Sigmar $1^{-/-}$skeletal muscles is similar to that observed in tubular aggregate myopathy [55-63], a rare myopathy characterized by a progressive decline in skeletal muscle function with increased weakness, cramps, and pain [55]. Structural abnormalities of mitochondria [5659], and defects in mitochondrial function [58, 60, 61], had been reported in myopathies with tubular aggregates [56-64]. Studies have shown either gain or loss of function mutations in STIM1, Orai1, and CASQ1 gene modulating the cellular calcium levels in either direction to cause the formation of tubular aggregates $[43,44,65,66]$. Though studies demonstrated, tubular aggregates were formed by the accumulation of sarcoplasmic reticulum (SR)-derived tubules, the molecular mechanism remains unknown.

In conclusion, we reported, for the first time, the physiological function of Sigmar1 in skeletal muscles using Sigmar1 null mice showing interesting phenotypes including increased central nuclei number, deposition of tubular aggregates, derangement in dystrophin localization, myofiber type switch from type I to type II and vice versa depending on the muscle type, and reduced endurance and exercise tolerance. A significant limitation of our study is the use of global knockout mice, where we cannot rule out a possible contribution to the observed skeletal muscle pathology due to Sigmar1 ablation in other organs. Though the muscle dysfunctions, including tubular aggregate and dystrophin delocalization, are unique to skeletal muscle pathology, future studies are required to recapitulate these data using a skeletal muscle fiber-specific knockout mouse. Our results further explain the skeletal muscle pathology observed in Sigmar1 mutation-bearing patients may elicit direct effects of dysfunctional Sigmar1 in myofibers and require further studies to demonstrate the molecular mechanism.

\section{Methods}

\section{Animals}

We used 9-10 months of age gender, and littermate control global Sigmar1 knockout (Sigmar1 $1^{-/}$) mice as previously reported $[8,9]$ and wildtype mice on the C57BL/ 6 background. The mice were accommodated in a well-controlled environment in cages with ad libitum water and a regular chow diet following 12-hour light-dark cycle. Equal numbers of both male and female mice consisting of Sigmar ${ }^{-1}$ and wildtype (WT) mice were used for the experiments. The animal handling procedures were in accordance with the Guide for Care and Use of Laboratory Animals, with the protocols being approved by the ACUC Committee of LSU Health Sciences Center-Shreveport. The animals were cared for according to the National Institute of Health guidelines for the care and use of the laboratory animals. 


\section{Muscle isolation and morphometry}

Mice from both the groups (WT and Sigmar1 $1^{--}$) were subjected to isofluorane-mediated anesthesia. Five different muscles, namely gastrocnemius (Gastro), quadriceps (Quad), soleus (Sol), tibialis anterior (TA), and extensor digitorum longus (EDL) were isolated from both limbs of each group of mice and were processed according to the experimental requirements. For morphometric analysis, the isolated muscles were washed with 1X PBS to remove any fur and any fat or bloodstains. The tissues were soaked using clean paper towels, and the wet tissue weights were taken using the OHAUS ${ }^{\circ}$ electronic balance machine (NJ, USA).

\section{Protein isolation and Western blotting}

Total proteins were prepared from all five different muscles isolated from both WT and Sigmar $1^{1-}$ mice. The isolated muscles were lysed with Cell Lytic M (Sigma-Aldrich) lysis buffer supplemented with Complete Protease Inhibitor Cocktail (Roche) as described previously. All the muscle sections were homogenized twice using bead homogenizer followed by sonication. The muscle homogenates were then centrifuged at $12,000 \mathrm{~g}$ for 15 minutes to sediment the insoluble cell debris. The protein concentration of the muscle homogenate was measured using the Bradford protocol/reagent (Bio-Rad) relative to a BSA standard curve (Bio-Rad). Protein samples were then separated on SDS-PAGE using precast $5 \%-12 \%$ gel Criterion gels (Bio-Rad) and transferred to PVDF membranes (Bio-Rad). Membranes were blocked for 1 hour in $5 \%$ non-fat dried milk and exposed to primary antibodies overnight. The following primary antibodies were used for immunoblotting: Sigmar1 (1:1000, 61994, Cell Signalling), GAPDH (1:10000, MAB374, EMD Millipore), and $\beta$-actin (1:1000, sc-47778, Santacruz). Subsequently, membranes were washed, incubated with alkaline phosphatase-conjugated secondary antibodies (Jackson ImmunoResearch Laboratories, Inc.), developed with ECF reagent (Amersham), and imaged by using Chemidoc $^{T M}$ Touch Imaging System (BioRad). Densitometric analysis of the protein bands on the scanned image was done using ImageJ software ( $\mathrm{NIH}$, Bethesda, MD).

\section{WGA staining}

Wheat germ agglutinin (WGA) staining was used to measure the cross-sectional area of the myofibers of different muscles as described previously $[9,67]$. Briefly, all five different muscles isolated from WT and Sigmar $\%$ mice were fixed in $10 \%$ buffered formalin and embedded in paraffin. These paraffin-embedded blocks were cut into $5 \mu \mathrm{M}$ serial sections, de-paraffinized, and hydrated followed by antigen retrieval by boiling at $100^{\circ} \mathrm{C}$ in $10 \mathrm{mmol} / \mathrm{L}$ sodium citrate buffer $(\mathrm{pH} 6.0)$. Subsequently, the sections were blocked for 1 hour with blocking solution ( $1 \%$ BSA, $0.1 \%$ cold water fish skin gelatin, and $1 \%$ Tween 20 in PBS) at room temperature and then, incubated with Alexa Fluor 488 wheat germ agglutinin $(5 \mu \mathrm{g} / \mathrm{mL}$, Invitrogen) for $1 \mathrm{hr}$ at room temperature, and nuclei were counterstained with 4'-6diamidino-2-phenylindole (DAPI, Invitrogen) for 5 minutes. The stained slides were washed with 1X PBS and then mounted using Vectashield Hardset antifade mounting media for fluorescence (Vector Laboratories). The stained sections were then subsequently observed using Nikon A1R high-resolution 
confocal microscope (Nikon Instruments Inc., Melville, NY) and imaged with Nikon NIS elements software (v4.13.04) with a 20x objective lens. All image acquisition was performed in an investigator-blinded manner. 3000-4000 myocytes from 4-28 images for each muscle from each mice group were blindly selected and were used to calculate the average cross-sectional area of the muscle fiber using ImageJ software (NIH, Bethesda, MD). Central nuclei were quantified by counting the number of DAPI positive stains inside the myocyte using ImageJ software (NIH, Bethesda, MD).

\section{Immunostaining}

We used $5 \mu \mathrm{m}$ serial cut sections from paraffin-embedded blocks of quadriceps muscle from WT mice to detect the presence of Sigmar1 in muscle sections as described previously. Briefly, the muscle sections were deparaffinized, hydrated, and subjected to antigen retrieval by boiling at $100^{\circ} \mathrm{C}$ in $10 \mathrm{mmol} / \mathrm{L}$ sodium citrate buffer $(\mathrm{pH} 6.0)$ for 30 minutes. The sections were treated with enhancer for 30 minutes following $1 \mathrm{xPBS}$ wash. The enhanced tissue sections were then washed and blocked with blocking buffer (1\% BSA, $0.1 \%$ cold water fish skin gelatin, and 1\%Tween 20 in PBS) for 1 hour at room temperature. The blocked sections were then incubated with primary antibodies overnight at $4^{0} \mathrm{C}$ in a humidified chamber followed by Alexa Fluor conjugated dyes (1:100) for 1 hour and 30 minutes at room temperature in a humidified chamber. Subsequently, the sections were incubated with either second primary antibodies (1 hr 30 minutes) or WGA ( $5 \mu \mathrm{g} / \mathrm{mL}$, Invitrogen) $(1 \mathrm{hr})$ at room temperature. Following second antibody incubation, the sections were incubated with Alexa Fluor conjugated secondary antibody (1:100) for 1 hour 30 minutes. All the stained sections exposed to 4'-6-diamidino-2-phenylindole (DAPI, Invitrogen) for 5 minutes at room temperature and mounted with Vectashield Hardset antifade mounting media for fluorescence (Vector Laboratories). The stained sections were then observed using Nikon A1R high-resolution confocal microscope (Nikon Instruments Inc., Melville, NY) and imaged with Nikon NIS elements software (v4.13.04) with a 20x objective lens. All image acquisition was performed in an investigator-blinded manner. The primary antibodies used were Sigmar1 N-terminal antibody (1:100, OAAB01426, Aviva), Sigmar1 C-terminal antibody (1:100, 61994, Cell Signalling), Dystrophin (1:100, D8168, Sigma-Aldrich), a-sarcomeric actinin (skeletal muscle marker) (1:100, A7811, Sigma-Aldrich), Type 1 myosin heavy chain (Type I or oxidative muscle marker) (1:100, BA-F8, DSHB) and OxPhos (mitochondrial marker) (1:100, ab110413, Abcam). The secondary antibodies used were Alexa Fluor 488 (A11034) and Alexa Fluor 568 (A11031) from Invitrogen. For type I stained myocytes, at least 1000 myocytes were measured per group for each muscle. The images were blindly selected, and quantifications were done using Image J software ( $\mathrm{NIH}$, Bethesda, MD).

\section{Histological Analyses}

Paraffin-embedded blocks for all five different muscles collected from WT and Sigmar ${ }^{-1}$ mice were cut into serial sections of $5 \mu \mathrm{m}$. The sections were deparaffinized, hydrated, and stained with PicroSirius red and Masson's Trichrome as previously described, and hematoxylin and eosin (H\&E staining kit, $\mathrm{H} 3502$, Vector Laboratories) as per the manufacturer's protocol. The stained sections were imaged in an invigilator-blinded manner using Olympus BX40 microscope in bright field mode with $20 x$ objective. The 
Collagen deposition and fibrosis in the muscle sections were quantified using NIH Image $\mathrm{J}$ software as described previously [9]. Briefly, the quantification for collagen deposition was done by quantitating the red-stained area and non-myocyte area from each section using color-based thresholding. Fibrosis quantification was done by determining blue-stained area and non-myocyte area from each section using color-based thresholding. The percent of collagen deposition and total fibrosis area was calculated as a percentage of the red-stained area and blue-stained area, respectively, to the total area for each section. Hematoxylin and Eosin staining was used to show the presence of central nuclei in the histological sections of different muscles from WT and Sigmar $1^{-/-}$mice. 4-12 high magnification 20x images were used for quantification per muscle in each group for each mouse.

\section{Transmission Electron Microscopy}

Both WT and Sigmar1 ${ }^{-/}$mice were anesthetized with isoflurane, and all five muscles (Gastro, Quad, Sol, TA, and EDL) were collected and cut in small cubes of $1 \mathrm{~mm}^{3}$. The cut sections were fixed overnight in $3 \%$ glutaraldehyde in $0.1 \mathrm{M}$ sodium cacodylate buffer followed by post-fixation with $1 \%$ osmium tetroxide $\left(\mathrm{OsO}_{4}\right)$ and counterstain with uranyl acetate and lead salts. The sections were embedded using low viscosity epoxy resins. The thin cut sections were imaged in an investigator-blinded manner using JEOL JEM-1400 transmission electron microscope (JEOL, Peabody, MA) with Advanced Microscopy Techniques (AMT) digital camera (Woburn, MA).

\section{Muscle exercise tolerance and endurance capacity}

To assess endurance capacity and exercise tolerance of skeletal muscles, we subjected the mice to grip strength test and treadmill exercise, respectively. Endurance of the skeletal muscle was assessed by measuring forelimb grip strength as described in $[68,69]$ using an equal number of mice $(n=9)$ from both Wt and Sigmar1\% groups. Briefly, the mice were acclimated to the experiment room for at least 10 minutes. For the force measurement, the mice were placed on the mesh grid attached to the grip strength meter (1027SM, Columbus Instruments, $\mathrm{OH}$ ), allowing them to hold the grid using their forelimbs. Keeping the body of the mice parallel to the grid, they were slowly pulled away from the grid by holding the tail. The tension generated by the mice at the release of the grid was recorded by the gauge. The mice were allowed to rest for 1 minute and were subjected to another trial. Each mouse went for 5 trials. The data were recorded and analyzed using the Grip Strength Meter (GSM) software provided by the manufacturer.

An equal number of mice $(n=9)$ from both $W t$ and Sigmar1\%- groups were subjected to graded maximal exercise testing as described in [70]. Briefly, the mice were acclimated to the treadmill (OxyletPro, Panlab, Harvard Apparatus) using the condition described in Table 2 with an activated shock of $2 \mathrm{~mA}$. The acclimation of mice included three training sessions with 60 hours of recovery time followed by rest for one week. Following rest for one week, the mice were subjected to experimental treadmill exercise starting with the acclimation of mice in the motionless treadmill for 3 minutes followed by activation of shock and gradual escalation in speed and inclination as shown in Table 3 until exhaustion. Exhaustion criteria 
were defined by mice spending more than 5 seconds on the shock grid or getting more than 10 shocks. The data were analyzed for exercise tolerance using Metabolism V3.0 software (Harvard apparatus).

Table 1 is not available in this version.

Table 2: Acclimation (at 0\% inclination grade):

\begin{tabular}{|c|c|}
\hline Speed (m/min) & Duration (min) \\
\hline 0 & 3 \\
\hline 6 & 5 \\
\hline 9 & 2 \\
\hline 12 & 2 \\
\hline
\end{tabular}

Table 3: Treadmill exercise:

\begin{tabular}{|c|c|c|}
\hline Speed (m/min) & Duration (min) & Inclination (\% grade or degrees) \\
\hline 0 & 3 & 0 \\
\hline 6 & 2 & 0 \\
\hline 9 & 2 & 5 \\
\hline 12 & 2 & 10 \\
\hline 15 & 2 & 15 \\
\hline 18 & 1 & 15 \\
\hline 21 & 1 & 15 \\
\hline 23 & 1 & 15 \\
\hline 24 & 1 & 15 \\
\hline+1 until exhaustion & 1 & 15 \\
\hline
\end{tabular}

\section{Statistics and Reproducibility}

All in vivo studies were performed where investigators were blinded with respect to the mouse groups. We used a numerical ear tagging system for unbiased data collection. For all imaging studies, paraffin blocks, slides, and acquired microscopic images were alphanumerically labeled. Following the completion of the study, individual mouse ID and image ID numbers were cross-referenced with treatment to permit analysis. All statistical analyses were conducted in GraphPad Prism software (v8.4.1, La Jolla, $\mathrm{CA})$. Data were presented in graphs showing median and interquartile ranges. Two-tailed, unpaired t-test (for 2 groups) were used, followed by Tukey's multiple comparisons post hoc test. $P$ values less than 0.05 (95\% confidence interval) were considered significant.

\section{Reporting summary}

Further information on research design is available in the Nature Research Reporting Summary linked to this article.

\section{Declarations}

Data availability 
Source data for all graphs in this article are included in Supplementary Data 1. Uncropped images for all Western blots in this article are included in the Supplementary Information. Other information and data in this study are available from the corresponding author on reasonable request.

\section{Acknowledgments}

This work was supported by the National Institutes of Health grants: HL122354 and HL145753 to M. S. B; LSUHSC-S CCDS Finish Line Award, COVID-19 Research Award, LARC Research Award to M. S. B; P20GM121307 to C.G.K.; NIH R01 HL098435, HL133497, and HL141155 to A.W.O; LSUHSC-S Malcolm Feist Cardiovascular and AHA Postdoctoral Fellowship to C.S.A. (20POST35210789); AHA Postdoctoral Fellowship to S.A.; and LSUHSC-S Malcolm Feist Pre-doctoral Fellowship to R.A.

\section{Author contributions}

R.A., C.S.A., and M.S.B. conceptualized the study; R.A., C.S.A., and M.S.B. designed the experiments; R.A., C.S.A., S.A., N.S.R, M.M., and S.N. performed all experiments and participated in analyses; B.H. and J.K. performed electron microscopic experiments; M.A.N.B. performed statistical analysis; C.G.K., and A.W.O., contributed to analytic tools and reagents; R.A., C.S.A., and M.S.B. wrote the manuscript, and all of the authors have read, edited, and approved the paper.

\section{Competing interests}

The authors declare no competing interests.

\section{References}

1. Bhuiyan, M.S. and K. Fukunaga, Stimulation of Sigma-1 receptor signaling by dehydroepiandrosterone ameliorates pressure overload-induced hypertrophy and dysfunctions in ovariectomized rats. Expert Opinion on Therapeutic Targets, 2009. 13(11): p. 1253-1265.

2. Bhuiyan, S. and K. Fukunaga, Stimulation of Sigma-1 receptor by dehydroepiandrosterone ameliorates hypertension-induced kidney hypertrophy in ovariectomized rats. Experimental Biology and Medicine, 2010. 235(3): p. 356-364.

3. Bhuiyan, M.S., H. Tagashira, and K. Fukunaga, Dehydroepiandrosterone-Mediated Stimulation of Sigma-1 Receptor Activates Akt-eNOS Signaling in the Thoracic Aorta of Ovariectomized Rats with Abdominal Aortic Banding. Cardiovascular Therapeutics, 2011. 29(4): p. 219-230.

4. Klouz, A., et al., Evidence for sigma-1-like receptors in isolated rat liver mitochondrial membranes. (0007-1188 (Print)).

5. Kekuda, R., et al., Cloning and Functional Expression of the Human Type 1 Sigma Receptor (hSigmaR1). Biochemical and Biophysical Research Communications, 1996. 229(2): p. 553-558.

6. Mei, J. and G.W. Pasternak, Molecular cloning and pharmacological characterization of the rat sigma 1 receptor11Abbreviations: DTG, N,N'-di(o-tolyl-guanidine); HA, hemagglutinin; $R T$-PCR, reverse 
transcriptase-polymerase chain reaction; RACE, rapid amplification of CDNA ends; and CHO, Chinese hamster ovary. Biochemical Pharmacology, 2001. 62(3): p. 349-355.

7. Soriani, O. and R. Rapetti-Mauss, Sigma 1 Receptor and Ion Channel Dynamics in Cancer, in Sigma Receptors: Their Role in Disease and as Therapeutic Targets, S.B. Smith and T.-P. Su, Editors. 2017, Springer International Publishing: Cham. p. 63-77.

8. Bernard-Marissal, N., et al., Dysfunction in endoplasmic reticulum-mitochondria crosstalk underlies SIGMAR1 loss of function mediated motor neuron degeneration. Brain, 2015. 138(4): p. 875-890.

9. Abdullah, C.S., et al., Cardiac Dysfunction in the Sigma 1 Receptor Knockout Mouse Associated With Impaired Mitochondrial Dynamics and Bioenergetics. Journal of the American Heart Association, 2018. 7(20): p. e009775.

10. Christ, M.G., et al., Sigma-1 Receptor Activation Induces Autophagy and Increases Proteostasis Capacity In Vitro and In Vivo. Cells, 2019. 8(3): p. 211.

11. Martin, W.R., et al., The effects of morphine-and nalorphine- like drugs in the nondependent and morphine-dependent chronic spinal dog. Journal of Pharmacology and Experimental Therapeutics, 1976. 197(3): p. 517-532.

12. Su, T.P., Evidence for sigma opioid receptor: binding of [3H]SKF-10047 to etorphine-inaccessible sites in guinea-pig brain. Journal of Pharmacology and Experimental Therapeutics, 1982. 223(2): p. 284290.

13. Loeffler, J.P., et al., The Role of Skeletal Muscle in Amyotrophic Lateral Sclerosis. Brain Pathol, 2016. 26(2): p. 227-36.

14. Al-Saif, A., F. Al-Mohanna, and S. Bohlega, A mutation in sigma-1 receptor causes juvenile amyotrophic lateral sclerosis. Annals of Neurology, 2011. 70(6): p. 913-919.

15. Watanabe, S., et al., Mitochondria-associated membrane collapse is a common pathomechanism in SIGMAR1- and SOD1-linked ALS. EMBO Molecular Medicine, 2016. 8(12): p. 1421-1437.

16. Li, X., et al., A SIGMAR1 splice-site mutation causes distal hereditary motor neuropathy. Neurology, 2015. 84(24): p. 2430-2437.

17. Gregianin, E., et al., Loss-of-function mutations in the SIGMAR1 gene cause distal hereditary motor neuropathy by impairing ER-mitochondria tethering and Ca2 + signalling. Human Molecular Genetics, 2016. 25(17): p. 3741-3753.

18. Horga, A., et al., <em> SIGMAR1</em> mutation associated with autosomal recessive Silver-like syndrome. Neurology, 2016. 87(15): p. 1607-1612.

19. Lee, J.J.Y., et al., Further Validation of the SIGMAR1 c.151 + 1G > T Mutation as Cause of Distal Hereditary Motor Neuropathy. Child Neurology Open, 2016. 3: p. $2329048 X 16669912$.

20. Almendra, L., et al., SIGMAR1 gene mutation causing Distal Hereditary Motor Neuropathy in a Portuguese family. Acta Myol, 2018. 37(1): p. 2-4.

21. Nandhagopal, R., et al., Recessive distal motor neuropathy with pyramidal signs in an Omani kindred: underlying novel mutation in the SIGMAR1 gene. Eur J Neurol, 2018. 25(2): p. 395-403. 
22. Couly, S., et al., Sigma-1 receptor is a key genetic modulator in amyotrophic lateral sclerosis. Hum Mol Genet, 2020. 29(4): p. 529-540.

23. Luty, A.A., et al., Sigma nonopioid intracellular receptor 1 mutations cause frontotemporal lobar degeneration-motor neuron disease. Annals of Neurology, 2010. 68(5): p. 639-649.

24. Langa, F., et al., Generation and phenotypic analysis of sigma receptor type I (sigma 1) knockout mice. Eur J Neurosci, 2003. 18(8): p. 2188-96.

25. Bernard-Marissal, N., et al., Dysfunction in endoplasmic reticulum-mitochondria crosstalk underlies SIGMAR1 loss of function mediated motor neuron degeneration. Brain, 2015. 138(Pt 4): p. 875-90.

26. Mavlyutov, T.A., et al., The sigma-1 receptor is enriched in postsynaptic sites of C-terminals in mouse motoneurons. An anatomical and behavioral study. Neuroscience, 2010. 167(2): p. 247-55.

27. Watanabe, S., et al., Mitochondria-associated membrane collapse is a common pathomechanism in SIGMAR1- and SOD1-linked ALS. EMBO Mol Med, 2016. 8(12): p. 1421-1437.

28. Sabino, V., et al., Sigma-1 receptor knockout mice display a depressive-like phenotype. Behav Brain Res, 2009. 198(2): p. 472-6.

29. Scott, W., J. Stevens, and S.A. Binder-Macleod, Human Skeletal Muscle Fiber Type Classifications. Physical Therapy, 2001. 81(11): p. 1810-1816.

30. Guy, P.S. and D.H. Snow, Skeletal muscle fibre composition in the dog and its relationship to athletic ability. Research in Veterinary Science, 1981. 31(2): p. 244-248.

31. Mazzotti, A.L. and D. Coletti, The Need for a Consensus on the Locution "Central Nuclei" in Striated Muscle Myopathies. Frontiers in Physiology, 2016. 7(577).

32. Gutiérrez, J.i.a., C.L. Ownby, and G.V. Odell, Skeletal muscle regeneration after myonecrosis induced by crude venom and a myotoxin from the snake Bothrops asper (Fer-de-Lance). Toxicon, 1984. 22(5): p. 719-731.

33. Folker, E. and M. Baylies, Nuclear positioning in muscle development and disease. Frontiers in Physiology, 2013. 4(363).

34. Cadot, B., V. Gache, and E.R. Gomes, Moving and positioning the nucleus in skeletal muscle - one step at a time. Nucleus, 2015. 6(5): p. 373-381.

35. Boyer, J.G., et al., ERK1/2 signaling induces skeletal muscle slow fiber-type switching and reduces muscular dystrophy disease severity. JCI Insight, 2019. 4(10).

36. Jennifer, G. and S. Masatoshi, Skeletal Muscle Fiber Types in Neuromuscular Diseases. 2018.

37. Izumi, Y., et al., Compound heterozygote mutations in the SIGMAR1 gene in an oldest-old patient with amyotrophic lateral sclerosis. Geriatrics \& Gerontology International, 2018. 18(10): p. 1519-1520.

38. Almendra, L., et al., SIGMAR1 gene mutation causing Distal Hereditary Motor Neuropathy in a Portuguese family. Acta myologica: myopathies and cardiomyopathies : official journal of the Mediterranean Society of Myology, 2018. 37(1): p. 2-4.

39. Christodoulou, K., et al., A novel form of distal hereditary motor neuronopathy maps to chromosome 9p21.1-p12. Annals of Neurology, 2000. 48(6): p. 877-884. 
40. Belzil, V.V., et al., Genetic analysis of SIGMAR1 as a cause of familial ALS with dementia. European Journal of Human Genetics, 2013. 21(2): p. 237-239.

41. Kim, H.-J., et al., Mutations in UBQLN2 and SIGMAR1 genes are rare in Korean patients with amyotrophic lateral sclerosis. Neurobiology of Aging, 2014. 35(8): p. 1957.e7-1957.e8.

42. Ullah, M.I., et al., In silico analysis of SIGMAR1 variant (rs4879809) segregating in a consanguineous Pakistani family showing amyotrophic lateral sclerosis without frontotemporal lobar dementia. neurogenetics, 2015. 16(4): p. 299-306.

43. Lee, J.-M. and S. Noguchi, Calcium Dyshomeostasis in Tubular Aggregate Myopathy. International Journal of Molecular Sciences, 2016. 17(11): p. 1952.

44. Okuma, H., et al., Tubular aggregate myopathy caused by a novel mutation in the cytoplasmic domain of < em > STIM1</em>. Neurology Genetics, 2016. 2(1): p. e50.

45. Couly, S., et al., Knocking Out Sigma-1 Receptors Reveals Diverse Health Problems. Cellular and Molecular Neurobiology, 2020.

46. Pal, A., et al., The sigma-1 receptor protects against cellular oxidative stress and activates antioxidant response elements. European Journal of Pharmacology, 2012. 682(1): p. 12-20.

47. Abdullah, C.S., et al., Cardiac Dysfunction in the Sigma 1 Receptor Knockout Mouse Associated With Impaired Mitochondrial Dynamics and Bioenergetics. J Am Heart Assoc, 2018. 7(20): p. e009775.

48. Bhuiyan, M.S. and K. Fukunaga, Stimulation of sigma-1 receptor signaling by dehydroepiandrosterone ameliorates pressure overload-induced hypertrophy and dysfunctions in ovariectomized rats. Expert Opin Ther Targets, 2009. 13(11): p. 1253-65.

49. Bhuiyan, M.S. and K. Fukunaga, Targeting sigma-1 receptor signaling by endogenous ligands for cardioprotection. Expert Opin Ther Targets, 2011. 15(2): p. 145-55.

50. Bhuiyan, M.S., et al., Targeting sigma-1 receptor with fluvoxamine ameliorates pressure-overloadinduced hypertrophy and dysfunctions. Expert Opin Ther Targets, 2010. 14(10): p. 1009-22.

51. Tagashira, H., et al., Fluvoxamine rescues mitochondrial Ca2 + transport and ATP production through sigma(1)-receptor in hypertrophic cardiomyocytes. Life Sci, 2014. 95(2): p. 89-100.

52. Tagashira, H., et al., Sigma 1-receptor stimulation with fluvoxamine ameliorates transverse aortic constriction-induced myocardial hypertrophy and dysfunction in mice. Am J Physiol Heart Circ Physiol, 2010. 299(5): p. H1535-45.

53. Tagashira, H., et al., Methyl pyruvate rescues mitochondrial damage caused by SIGMAR1 mutation related to amyotrophic lateral sclerosis. Biochim Biophys Acta, 2014. 1840(12): p. 3320-34.

54. Staron, R.S., et al., Fiber type composition of the vastus lateralis muscle of young men and women. J Histochem Cytochem, 2000. 48(5): p. 623-9.

55. Walter, M.C., et al., 50 years to diagnosis: Autosomal dominant tubular aggregate myopathy caused by a novel STIM1 mutation. Neuromuscular Disorders, 2015. 25(7): p. 577-584.

56. Martin, J.J., C. Ceuterick, and G. Van Goethem, On a dominantly inherited myopathy with tubular aggregates. Neuromuscul Disord, 1997. 7(8): p. 512-20. 
57. Muller, H.D., et al., Dominantly inherited myopathy with novel tubular aggregates containing 1-21 tubulofilamentous structures. Acta Neuropathol, 2001. 102(1): p. 27-35.

58. Pierobon-Bormioli, S., et al., Familial neuromuscular disease with tubular aggregates. Muscle Nerve, 1985. 8(4): p. 291-8.

59. Rosenberg, N.L., H.E. Neville, and S.P. Ringel, Tubular aggregates. Their association with neuromuscular diseases, including the syndrome of myalgias/cramps. Arch Neurol, 1985. 42(10): p. 973-6.

60. Bendahan, D., et al., Magnetic resonance spectroscopy and histological study of tubular aggregates in a familial myopathy. J Neurol Sci, 1996. 139(1): p. 149-55.

61. Vielhaber, S., et al., Defective mitochondrial oxidative phosphorylation in myopathies with tubular aggregates originating from sarcoplasmic reticulum. J Neuropathol Exp Neurol, 2001. 60(11): p. $1032-40$.

62. Dobkin, B.H. and M.A. Verity, Familial neuromuscular disease with type 1 fiber hypoplasia, tubular aggregates, cardiomyopathy, and myasthenic features. Neurology, 1978. 28(11): p. 1135-40.

63. Rohkamm, R., et al., A dominantly inherited myopathy with excessive tubular aggregates. Neurology, 1983. 33(3): p. 331-6.

64. Sallinger, M., et al., Luminal STIM1 Mutants that Cause Tubular Aggregate Myopathy Promote Autophagic Processes. Int J Mol Sci, 2020. 21(12).

65. Böhm, J. and J. Laporte, Gain-of-function mutations in STIM1 and ORAl1 causing tubular aggregate myopathy and Stormorken syndrome. Cell Calcium, 2018. 76: p. 1-9.

66. Barone, V., et al., Identification and characterization of three novel mutations in the CASQ1 gene in four patients with tubular aggregate myopathy. Human Mutation, 2017. 38(12): p. 1761-1773.

67. Abdullah, C.S., et al., Methamphetamine induces cardiomyopathy by Sigmar1 inhibition-dependent impairment of mitochondrial dynamics and function. Communications Biology, 2020. 3(1): p. 682.

68. Castro, B. and S. Kuang, Evaluation of Muscle Performance in Mice by Treadmill Exhaustion Test and Whole-limb Grip Strength Assay. Bio-protocol, 2017. 7(8): p. e2237.


decline in skeletal muscle function in male mice. Scientific Reports, 2017. 7(1): p. 42323.

70. Petrosino, J.M., et al., Graded Maximal Exercise Testing to Assess Mouse Cardio-Metabolic Phenotypes. PLOS ONE, 2016. 11(2): p. e0148010.

\section{Supplemental Material}

Supplemental information is not available with this version of the text.

\section{Figures}





Overlay



$\alpha$-Sarcomeric actinin

DAPI
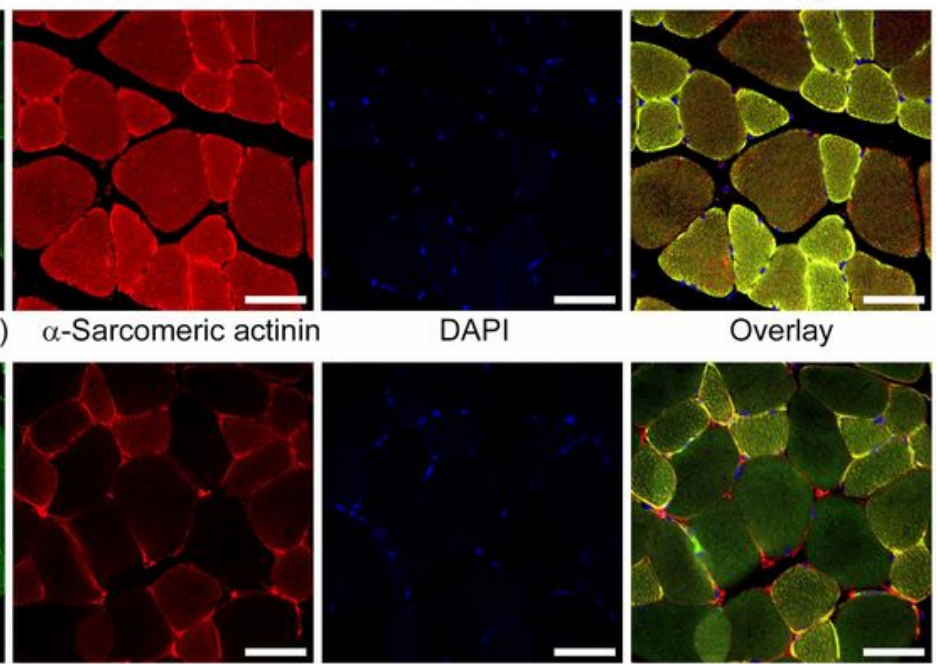

Overlay

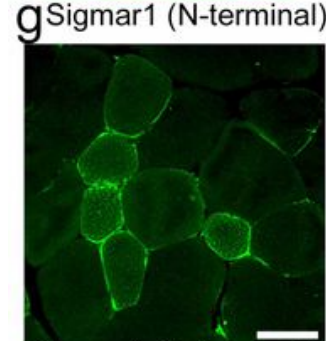

Sigmar1 ( $N$-terminal)



OXPHOS
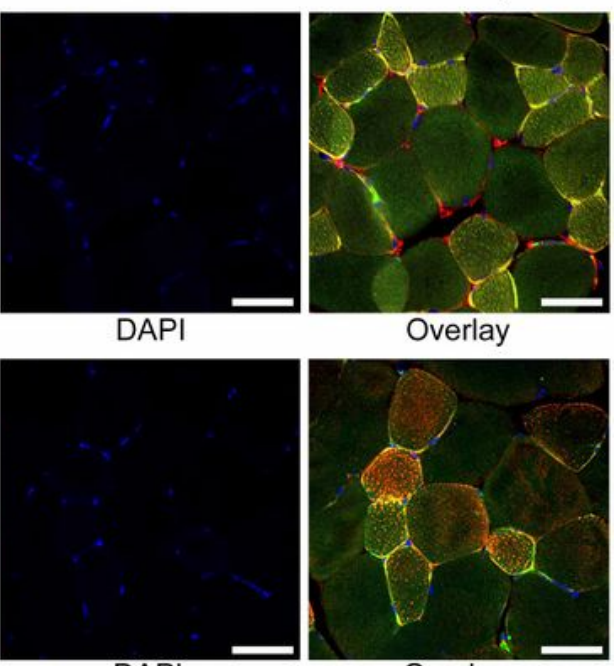

Overlay



Overlay

\section{Figure 1}

Expression and localization of Sigmar1 in the skeletal muscle. a Representative Western blot images demonstrating Sigmar1 protein level in the Gastrocnemius (Gastro), Quadriceps (Quad), Tibialis Anterior (TA), Soleus (Sol), and Extensor digitorum longus (EDL) muscles isolated from 9-10 months old Wt mice ( $n=4$ mice per skeletal muscle type). Cell lysates of Quad muscle isolated from Sigmar1 global knockout (Sigmar1-/-) and Sigmar1 overexpressing transgenic mice were used as a negative and positive control 
for Sigmar1 protein, respectively. $\beta$-actin was used to verify equal protein loading across the lanes. $b, c$ Bar graphs represent Sigmar1 protein and mRNA expression levels in the Gastro, Quad, TA, Sol, and EDL muscles extracted from 9-10 months old Wildtype mice. Dots in the bar graphs represent individual values quantified for muscle ( $n=8$ mice per muscle for Western blots, and $n=3$ mice per muscle for $m R N A$ ). Data are expressed as fold change of Gastro muscle and as mean \pm SEM d, e Representative immunofluorescence staining for Sigmar1 using antibodies directed against N-terminal and C-terminal of Sigmar1(green) and a-sarcomeric actinin (red) counterstaining showed distinct fluorescent puncta appeared at brighter fluorescence intensity in smaller diameter myofibers (presumably type I oxidative myofibers) compared to larger diameter myofibers (presumably type II glycolytic myofibers). $f, g$ Representative immunofluorescence staining for Sigmar1 (green) with type I myosin (red) and OXPHOS (red) counterstaining showed Sigmar1 fluorescent intensity relatively higher in type I myosin and mitochondria-rich myofibers, respectively. $\mathrm{d}$-g Quad muscles isolated from 9-10 months old Wt mice were used for immunostaining. Images are representative of two biological replicates (10-12 microscopic fields). DAPI staining was used to counterstain the nucleus. Scale bars $50 \mu \mathrm{m}$. 
a



b



Wt Quad

C



d



Wt Sol

e

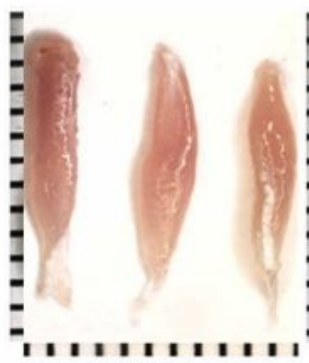

Wt EDL

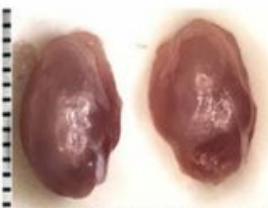

Sigmar1-\% Quad



Sigmar1 1 EDL



Wt Sigmar1 $\%$
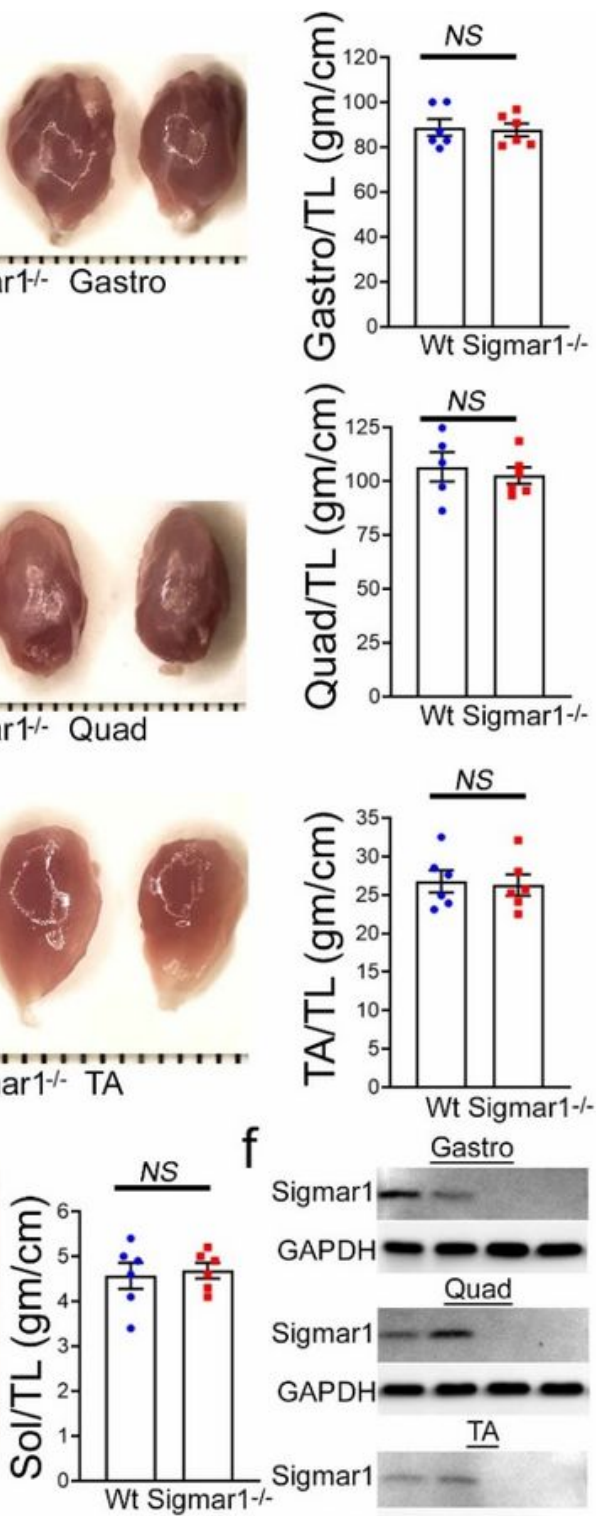

GAPDH - -

Sol

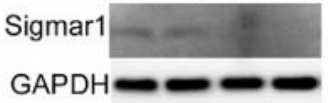

EDL



\section{Figure 2}

Morphometric analyses of skeletal muscles in Wt and Sigmar1-/- mice. a-e Left panels, gross morphological microscopic images of Gastrocnemius (Gastro), Quadriceps (Quad), Tibialis Anterior (TA), Soleus (Sol), and Extensor digitorum longus (EDL) muscles isolated from 9-10 months old Wt and Sigmar1-/- mice. Right panels, bar graphs representing muscle weight-to-tibia length (MW/TL) ratio of Gastro, Quad, TA, Sol, and EDL muscles. Dots in the bar graphs represent individual values quantified for 
each Wt and Sigmar1-/- mice ( $n=6$ mice for each muscle per genotype isolated from both of the limbs.). Data are expressed as mean \pm SEM. P values were determined by unpaired Student's t-test. NS, not significant. f Representative Western blot images of Sigmar1 protein in muscle tissue lysates isolated from Wt and Sigmar1-/- mice showing the absence of Sigmar1 in the muscles isolated from Sigmar1-/mice. $n=6$ mice for each muscle per genotype.



Figure 3

Altered myofiber size, presence of central nuclei, and fiber size distribution in Sigmar1-/- muscles compared to Wt muscles. Representative immunofluorescence images of wheat germ agglutinin (WGA) stained (green) a gastrocnemius (Gastro), e quadriceps (Quad), i tibialis anterior (TA), m soleus (Sol), and q extensor digitorum longus (EDL) muscle cross-sections from Wt and Sigmar1-/- mice, respectively. WGA was used to delineate myofiber boundaries, and DAPI was used to counterstain nuclei. Rectangle (white box) enclosed areas are presented in insets as digitally magnified areas to respective myocyte types, demonstrating the increased presence of central nuclei (white arrows) in Sigmar1-/- mice myocytes. Scale bars $50 \mu \mathrm{m}$. Box plots representing average myocyte cross-sectional areas ( $\mu \mathrm{m} 2$ ) for $b$ Gastro, $f$ Quad, j TA, n Sol, and r EDL muscle cross-sections from Wt and Sigmar1-/- mice, respectively. Gastro 
$(P<0.001)$ and Quad $(P<0.001)$ myofibers cross-sectional areas of Sigmar1-/- mice were significantly increased compared to Wt mice myofibers. Myofibers cross-sectional areas in Sigmar1-/- mice TA $(P<0.001)$ and EDL $(P<0.001)$ muscles were significantly reduced, whereas Sol muscle myofibers showed no changes in size compared to Wt mice myofibers. Bar graphs represent myofibers cross-sectional areas distribution frequency in c Gastro, g Quad, k TA, o Sol, and s EDL muscles of Wt and Sigmar1-/- mice. Cross-sectional areas were measured in 3000-4000 myocytes per muscle type in each mouse ( $n=3$ mice per genotype, 9-10 months old). Box plots representing the number of central nuclei counts per microscopic field in d Gastro, h Quad, I TA, $p$ Sol, and t EDL muscle cross-sections from Wt and Sigmar1-/- mice, respectively. Sigmar1-/- mice Gastro ( $P<0.05)$, Quad $(P<0.001)$, and TA $(P<0.01)$ muscles showed a significantly increased number of central nuclei compared to littermate Wt mice muscles. Central nuclei were counted in 4-28 high magnification (depending on the muscle size) microscopic fields (20x) per genotype for each mouse ( $n=3$ mice per genotype, 9-10 months old). Boxes represent interquartile ranges, lines represent medians, and whiskers represent ranges. $P$ values were determined by unpaired Student's t-test. NS, not significant. 
a
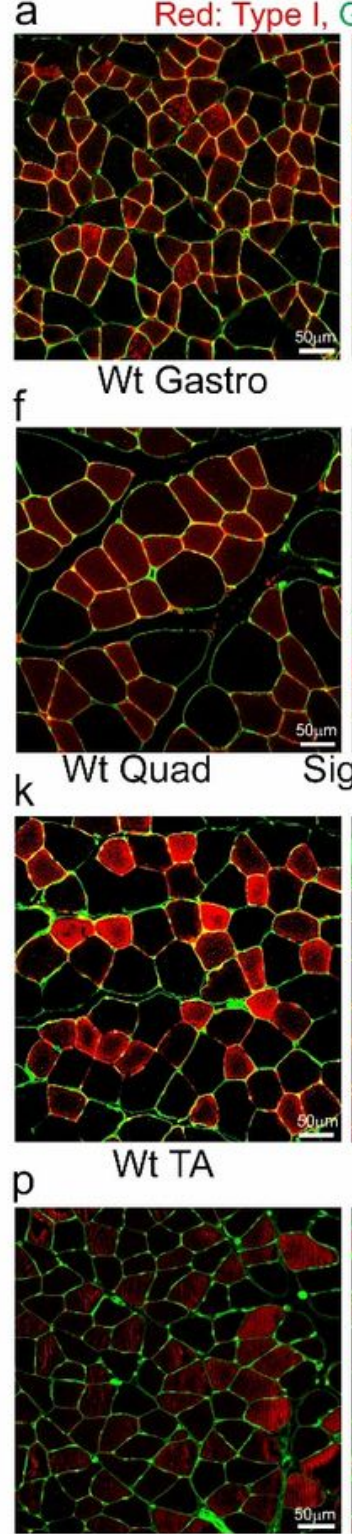

$\mathrm{u}$



Wt EDL



Sigmar1-1- Gastro



igmar1-1- Quad



Sigmar1 ${ }^{-/-}$TA

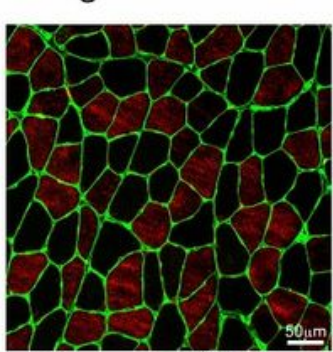

Sigmar1 ${ }^{-1-}$ Sol



Sigmar1 ${ }^{-1-}$ EDL
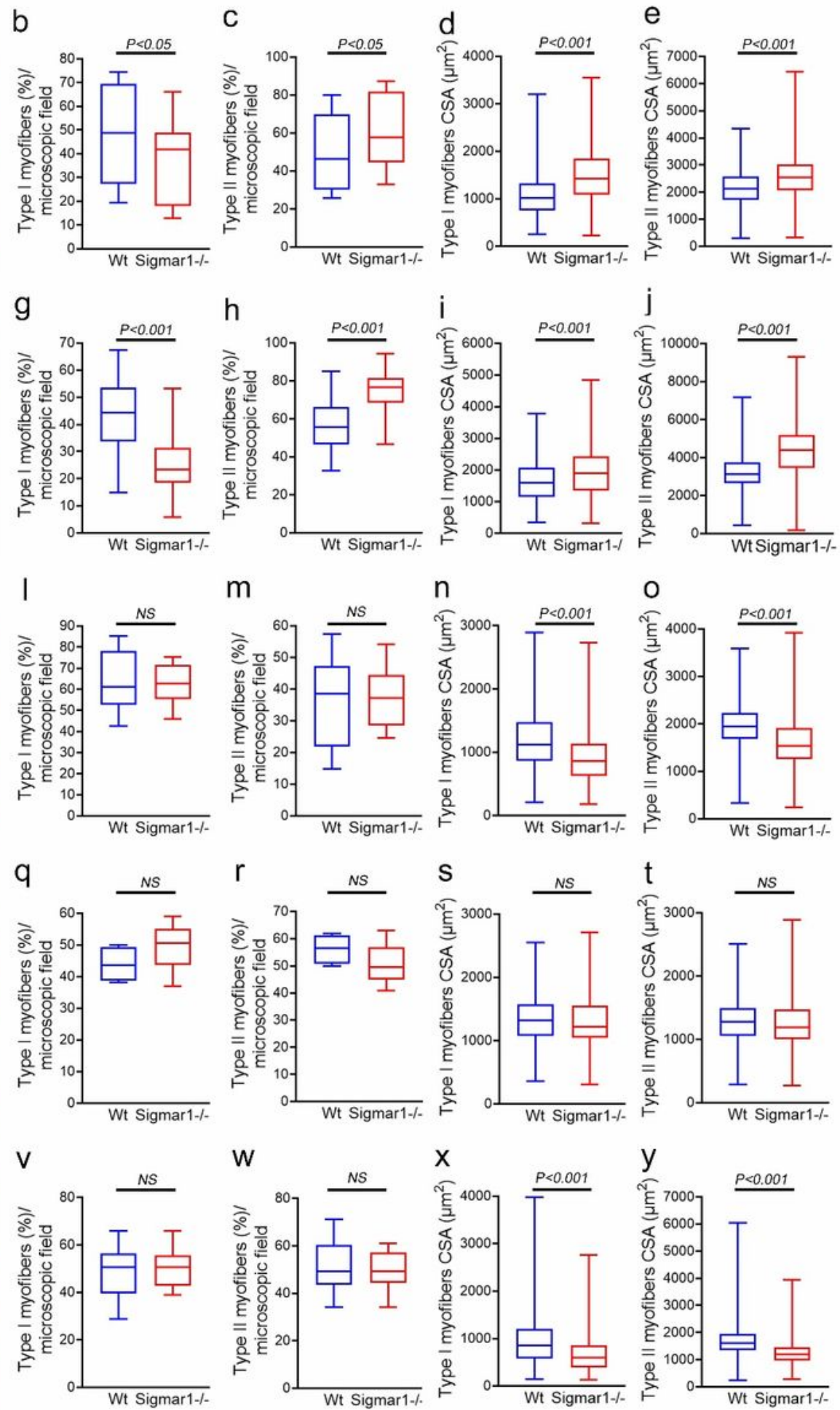

Figure 4

Sigmar1-/- mice showed altered myofiber isotypes and areas. a, f, k, p, u Representative immunofluorescence images for anti-Myosin type I (in red) and Wheat Germ Agglutinin (WGA; in green) staining in Gastro, Quad, TA, Sol, and EDL muscle sections from 9-10 months old Wt and Sigmar1-/mice. Scale bars $50 \mu \mathrm{m}$. b, c, g, h, I, m, q, r, v, w Box plots represent the percent of type I and type II myofibers per microscopic fields in Wt and Sigmar1-/- mice muscle sections. The absolute number of 
type I (myofibers stained in red) and type II (myofibers negative for anti-myosin Type I staining, thus, appeared as black) myofibers were quantified and expressed as a percent of total myofibers per microscopic field. Percent number of type I myofibers per microscopic field was significantly decreased in gastro and quad muscles in Sigmar1-/- mice compared to Wt mice. Whereas type II myofibers percent numbers were increased in Sigmar1-/- mice's gastro and quad muscles compared to Wt mice. We have not found any significant changes in the percent number of type I and type II fibers in TA, Sol, and EDL muscles between genotypes. The percent number of type I and type II muscle fiber per microscopic field were quantified in at least 4-10 microscopic fields per mice per muscle ( $n=3-5$ mice in each genotype). $d$, $\mathrm{e}, \mathrm{i}, \mathrm{j}, \mathrm{n}, \mathrm{o}, \mathrm{s}, \mathrm{t}, \mathrm{x}, \mathrm{y}$ Box plots represent cross-sectional areas ( $\mu \mathrm{m} 2)$ of type I and type II myofibers in Wt and Sigmar1-/- mice muscle sections. Cross-sectional areas were measured on WGA delineated individual myofiber membranes. Sigmar1-/- mice gastro and quad myofibers showed significantly increased areas of both type I and type II fibers than Wt mice. In contrast, both type I and type II myofiber areas were significantly reduced in TA and EDL muscles of Sigmar1-/-mice compared to Wt mice. There were no significant difference in myofiber areas in Sol between genotypes. Cross-sectional areas of type I and type II muscle fibers were measured on at least 1000 myocytes per muscle ( $n=3-5$ mice in each genotype). Boxes depict interquartile ranges, lines represent medians, and whiskers represent ranges. $P$ values were determined using a two-tailed unpaired Student's t-test. $\mathrm{P}<0.05$ between groups was considered statistically significant. NS, not significant. 


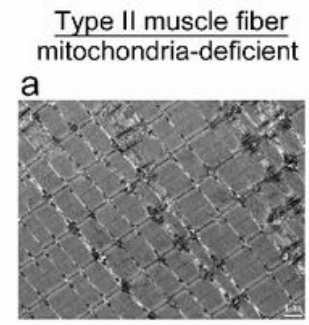

b
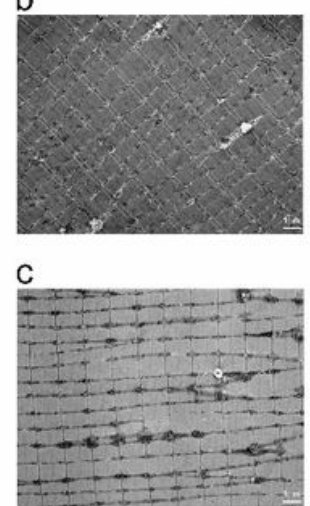

d



e

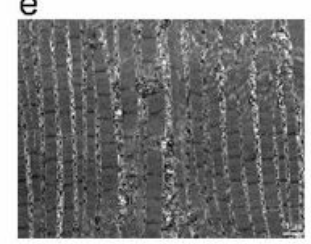

Type I muscle fiber

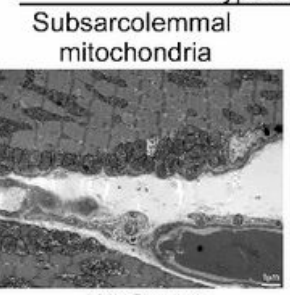

Wt Gastro

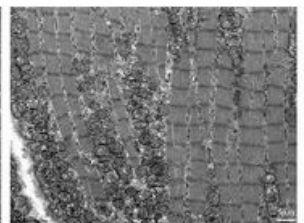

Wt Quad

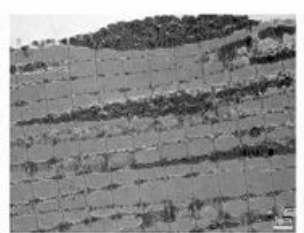

Wt TA



Wt Sol

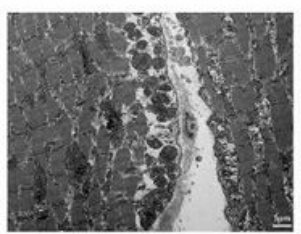

Wt EDL


Sigmar1 ${ }^{-/-}$Gastro
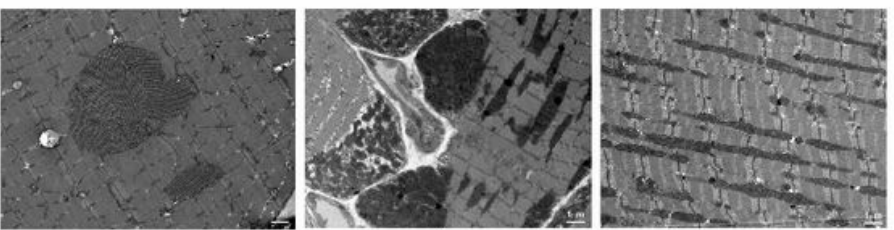

Sigmar1/- Quad
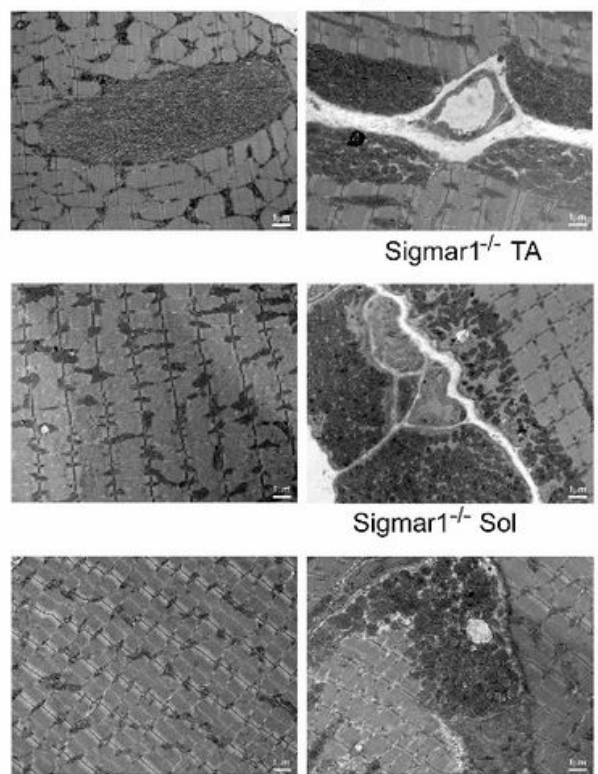

Sigmar1 ${ }^{-1-}$ TA



Sigmar $1^{-/-}$Sol
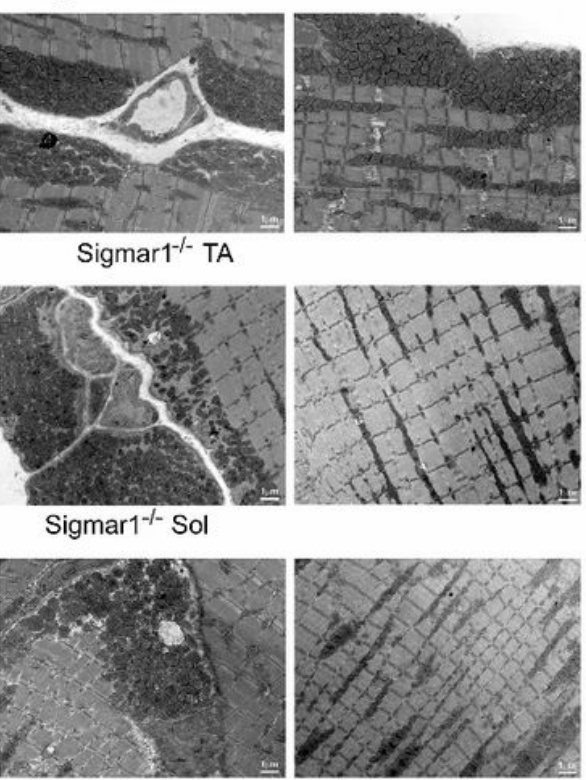

Sigmar1 ${ }^{-/-}$EDL

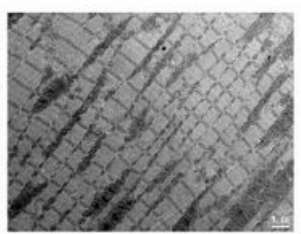

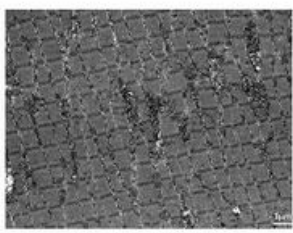

\section{Figure 5}

Tubular aggregates in mitochondria deficient type II myofibers in Sigmar1-/- mice skeletal muscles. a-e Representative transmission electron microscopy (TEM) images of a Gastrocnemius (Gastro), b Quadricep (Quad), c Tibialis Anterior (TA), d Soleus (Sol), and e Extensor digitorum longus (EDL) muscle sections from Wt and Sigmar1-/- mice. a-e Mitochondria deficient type II myofibers exhibit normal ultrastructure and sarcomere organization in Wt mice represented in all five muscle types. In contrast, Grasto, Quad, and TA muscles of Sigmar1-/- mice exhibit abnormal accumulation of crystalline-like tubular aggregates (honeycomb-shaped structures) between myofibrils and beneath the sarcolemma in type II myofibers. Normal subsarcolemmal and intermyofibrillar mitochondrial organization were observed in mitochondria-rich type I myofibers in all five muscle types of Wt mice. In contrast, abnormal and amorphous shaped mitochondria were observed in the muscles of Sigmar1-/- mice. All muscles examined were isolated from age-matched 9-10 months old littermate Wt and Sigmar1-/- mice ( $\mathrm{n}=3$ mice per genotype). Scale bars $1 \mu \mathrm{m}$. 


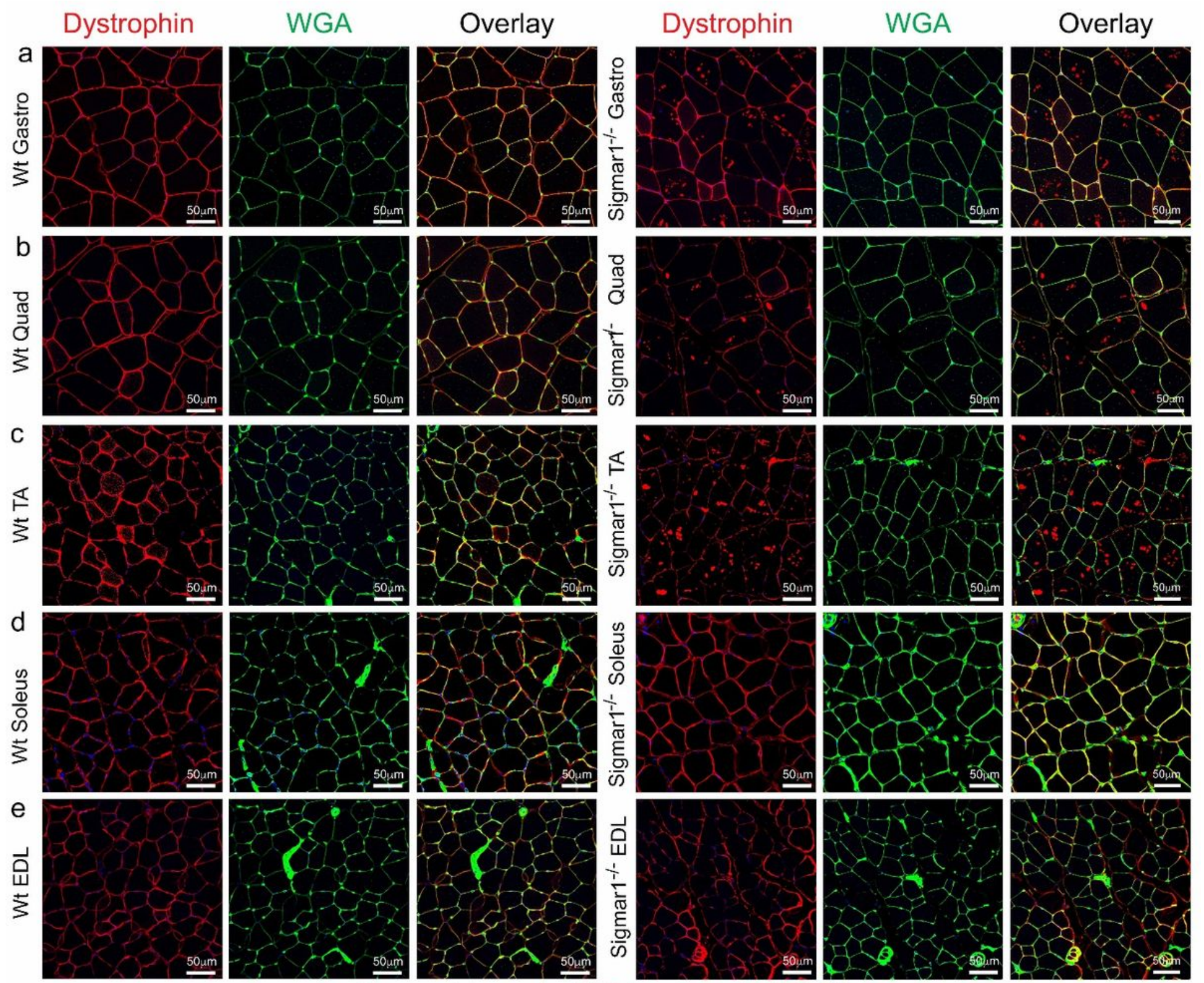

Dystrophin: red, WGA: green, DAPI: blue

\section{Figure 6}

Derangement of dystrophin localization in myofibers from Sigmar1-/- mice. a-e Representative immunofluorescence images of Wheat germ agglutinin (WGA; green) with dystrophin (red) counterstained a Gastrocnemius (Gastro), b Quadricep (Quad), c Tibialis Anterior (TA), d Soleus (Sol), and e Extensor digitorum longus (EDL) muscle sections from Wt and Sigmar1-/- mice. WGA staining was used to delineate myofiber boundaries. Anti-dystrophin staining (red) was conducted to delineate dystrophin localization in skeletal muscles. Dystrophin (red) localization appeared in sarcolemma as evident in colocalization with WGA (green) staining on myocytes membrane in Wt mice skeletal muscles. In contrast, aberrant dystrophin aggregates (in red) were detected intracellularly, particularly in myofibers with comparatively intermediate-to-large cross-sectional areas (presumably type II myofibers) in Gastro, Quad, and TA muscles of Sigmar1-/- mice. Representative images are from 3-10 high magnification microscopic fields (20x) ( $n=3$ mice per genotype at 9-10 months age). Scale bars $50 \mu \mathrm{m}$. 
a

b

$50 \mu \mathrm{m}$

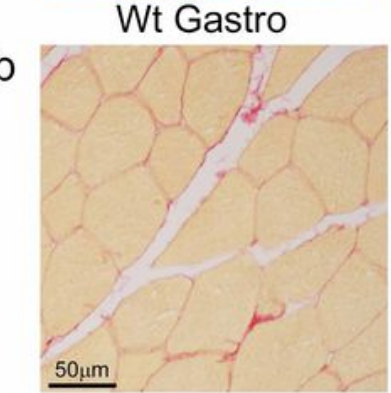

C

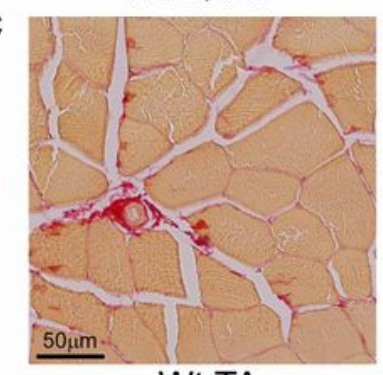

Wt TA

d

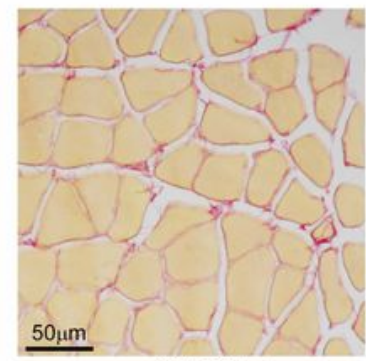

Wt Sol

e

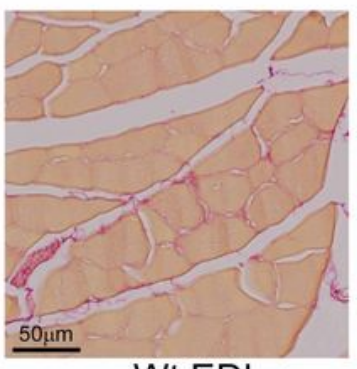

Wt EDL
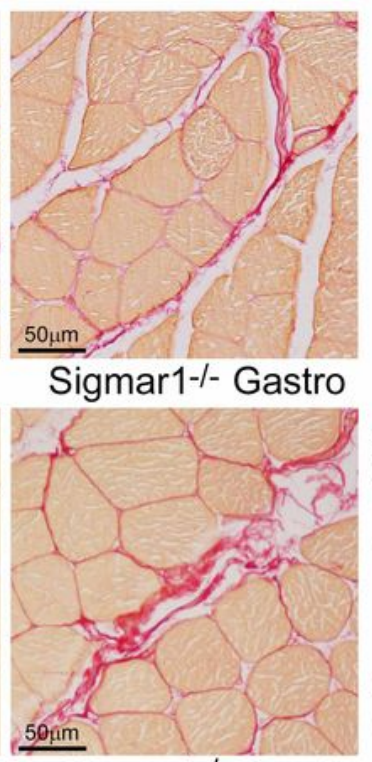

Sigmar1-/- Quad

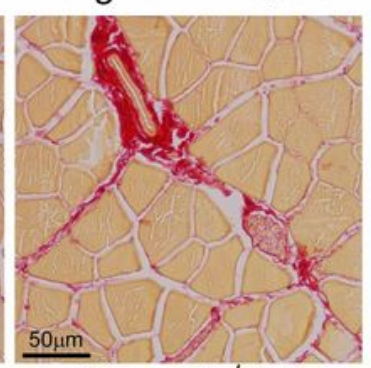

Sigmar1-/- TA

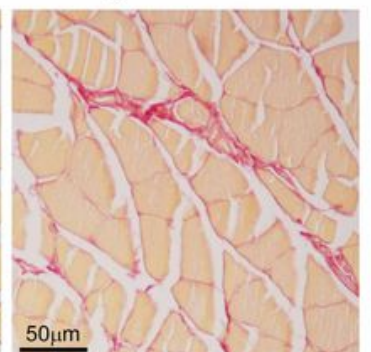

Sigmar1-- Sol

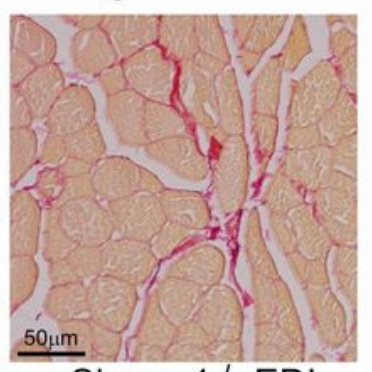

Sigmar1-/- EDL
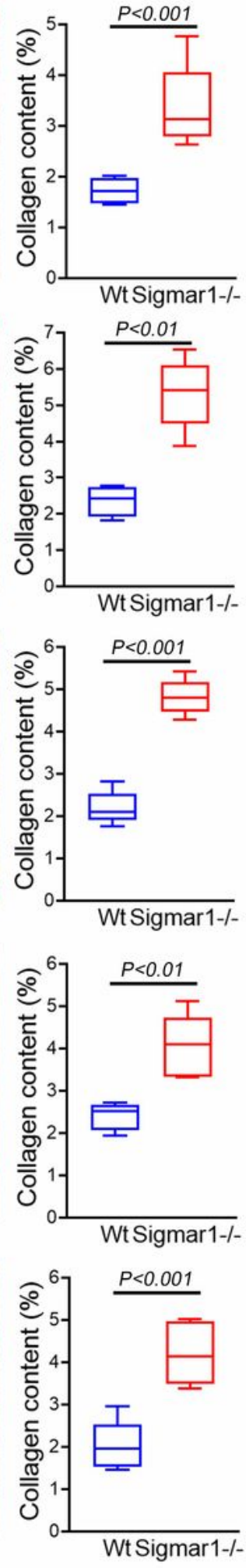

\section{Figure 7}

Increased collagen deposition in the muscles skeletal of Sigmar1-/- mice. a-e Left panels, representative images of Picro-Sirius red (PSR) stained histological cross-sections of a Gastrocnemius (Gastro), b Quadricep (Quad), c Tibialis Anterior (TA), d Soleus (Sol) and e Extensor digitorum longus (EDL) muscle sections from Wt and Sigmar1-/- mice. PSR staining revealed the presence of increased collagen deposition (stained in red) at all three connective tissue layers, including endomysium (surrounding the 
individual muscle), perimysium (enclosing a group of muscle fibers), and epimysium (surrounding the entire muscle segments) layers in Sigmar1-/- mice compared to Wt mice. Representative images are from 12 high magnification microscopic fields (20x) per mice muscle sections for Gastro, Quad, and TA muscles, and 5 high magnification microscopic fields (20x) for Sol and EDL muscles per mice $(n=4-5$ individual mice at 9-10 months age per genotype per muscle). Scale bars $50 \mu \mathrm{m}$. a-e Right panels, box plots represent percent collagen content measured in respective to PSR stained whole muscle crosssection areas in Wt and Sigmar1-/- mice skeletal muscles. Collagen content (\%) significantly increased in Sigmar1-/- mice muscle sections compared to Wt mice muscles. Quantifications of percent collagen areas were carried out on 12 high magnification microscopic fields (20x) per mice muscle sections for Gastro, Quad, and TA muscles, and on 5 high magnification microscopic fields (20x) for Soleus and EDL muscles per mice ( $n=4-5$ individual mice per genotype per muscle). $P$ values indicate statistical significance between Wt and Sigmar1-/- mice. Boxes represents interquartile ranges, lines represent medians, and whiskers represent ranges. $P$ values were determined by unpaired Student's t-test. 


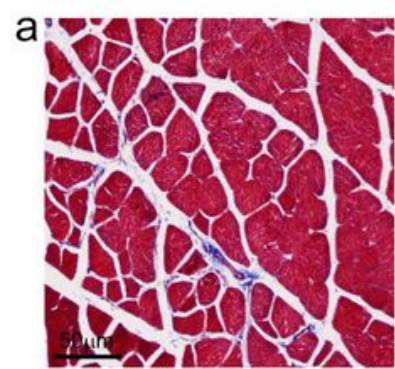

Wt Gastro

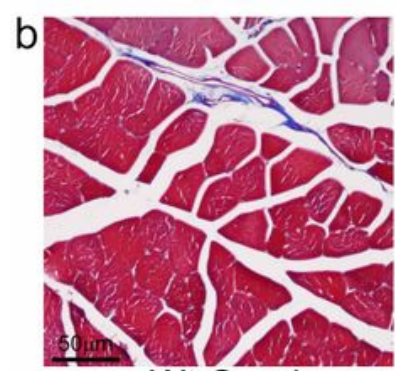

Wt Quad

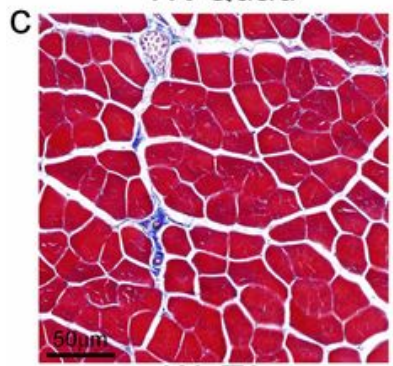

Wt TA

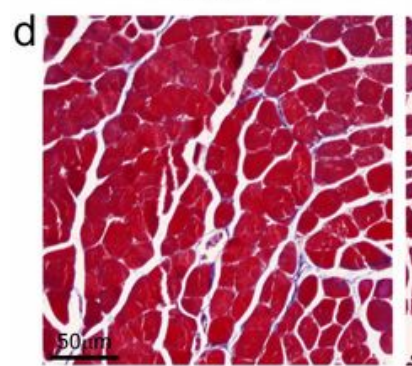

Wt Sol

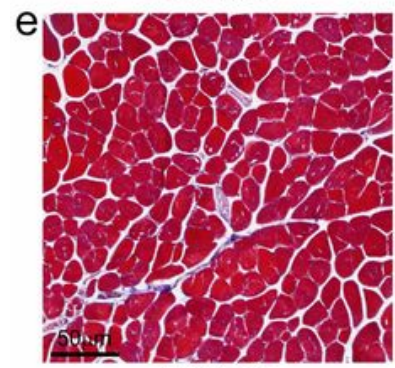

Wt EDL

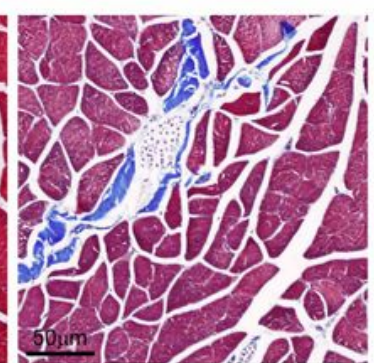

Sigmar 1-/- Gastro
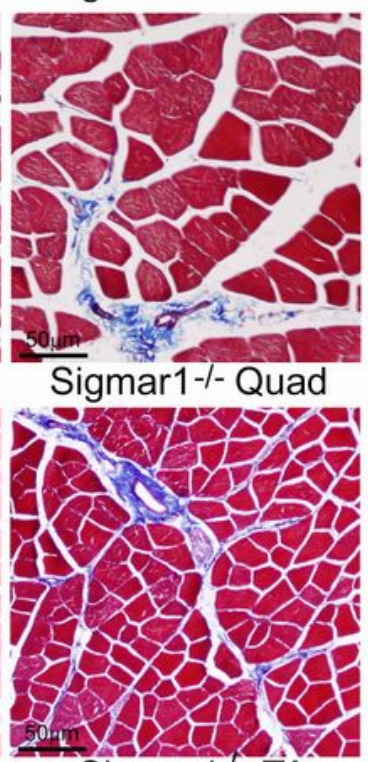

Sigmar1 ${ }^{-1-}$ TA
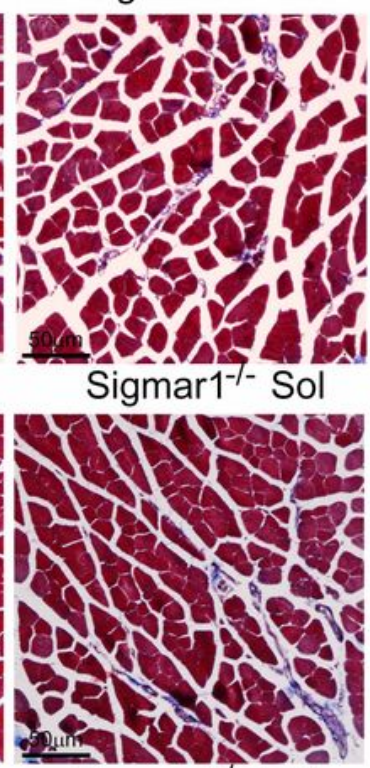

Sigmar1 ${ }^{-T-}$ EDL
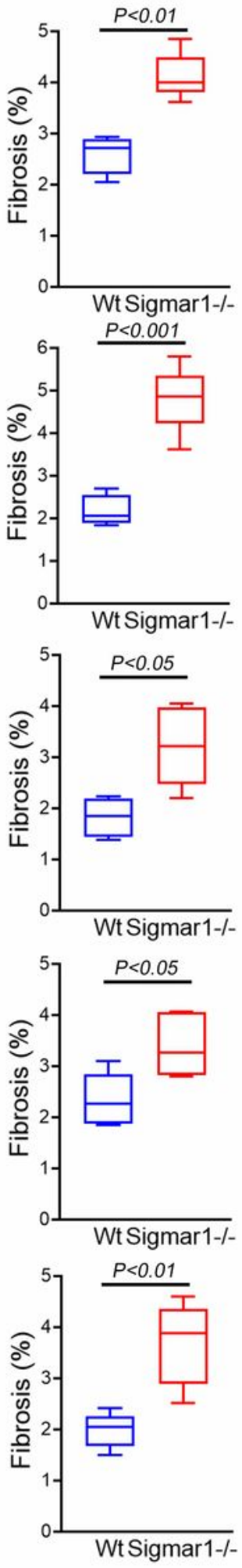

\section{Figure 8}

Increased fibrosis in the muscles skeletal of Sigmar1-/- mice compared to Wt mice. a-e Masson's Trichrome staining was conducted to assess interstitial fibrosis in muscle sections from Wt and Sigmar1-/- mice. Left panels, representative micrographs of Trichrome stained histological cross-sections of a Gastrocnemius (Gastro), b Quadricep (Quad), c Tibialis Anterior (TA), d Soleus (Sol), and e Extensor digitorum longus (EDL) muscle sections from Wt and Sigmar1-/- mice. Trichrome staining showed 
increased fibrosis areas (stained in blue) at connective tissue layers, including endomysium (enclosing individual myofiber), perimysium (enclosing a group of myofibers), and epimysium (surrounding an entire muscle segment) in Sigmar1-/- mice compared to Wt mice. Images represent 8-12 high magnification microscopic fields (20x) per mice muscle sections for Gastro, Quad, and TA muscles, and from 2-6 high magnification microscopic fields (20x) for Sol and EDL muscles per mice ( $\mathrm{n}=5$ individual mice at 9-10 months age per genotype per muscle). Scale bars $50 \mu \mathrm{m}$. a-e Right panels, box plots represent quantifications of percent fibrosis area corresponding to total muscle section areas in Trichrome stained whole muscle areas in Wt and Sigmar1-/- mice skeletal muscles. Percent fibrosis areas are significantly increased in Sigmar1-/- mice muscle sections compared to Wt mice muscles. Fibrosis areas (\%) were quantified on 8-12 high magnification microscopic fields (20x) per mice muscle sections for Gastro, Quad, and TA muscles, and on 2-6 high magnification microscopic fields (20x) for Sol and EDL muscles per mice ( $n=5$ individual mice per genotype per muscle). $P$ values indicate statistical significance between $\mathrm{Wt}$ and Sigmar1-/-mice. Boxes represent interquartile ranges, lines represent medians, and whiskers represent ranges. $P$ values were determined by unpaired Student's t-test. 


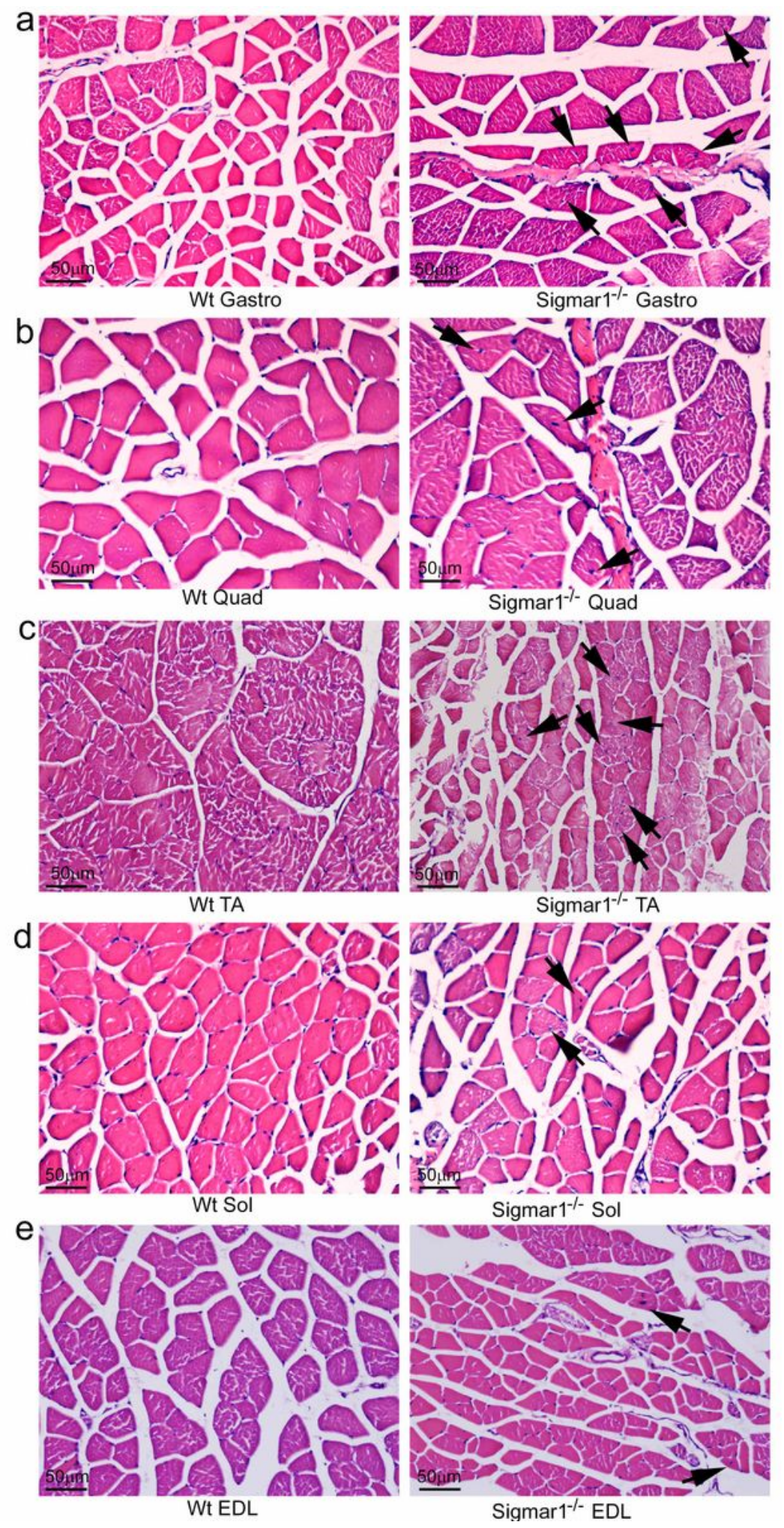

Figure 9

Histological H\&E staining in Wt and Sigmar1-/- mice skeletal muscle sections. Representative micrographs of H\&E stained cross-sections of a Gastrocnemius (Gastro), b Quadricep (Quad), c Tibialis Anterior (TA), d Soleus (Sol), and e Extensor digitorum longus (EDL) muscle sections from Wt and Sigmar1-/- mice. Sigmar1-/- mice muscles exhibit central nuclei (black arrows) in myofibers compared to peripheral nuclei in Wt mice muscle sections. An increase in interstitial eosinophilic collagen deposition 
(bright pink fibers) has also been observed in Sigmar1-/- mice muscle sections compared to Wt mice muscle sections. Representative images are from 8-12 high magnification microscopic fields (20x) per mice muscle sections for Gastro, Quad, and TA muscles, and from 2-6 high magnification microscopic fields (20x) for Sol and EDL muscles per mice ( $n=5$ individual mice at 9-10 months age per genotype per muscle). Scale bars $50 \mu \mathrm{m}$. Scale bars $50 \mu \mathrm{m}$.

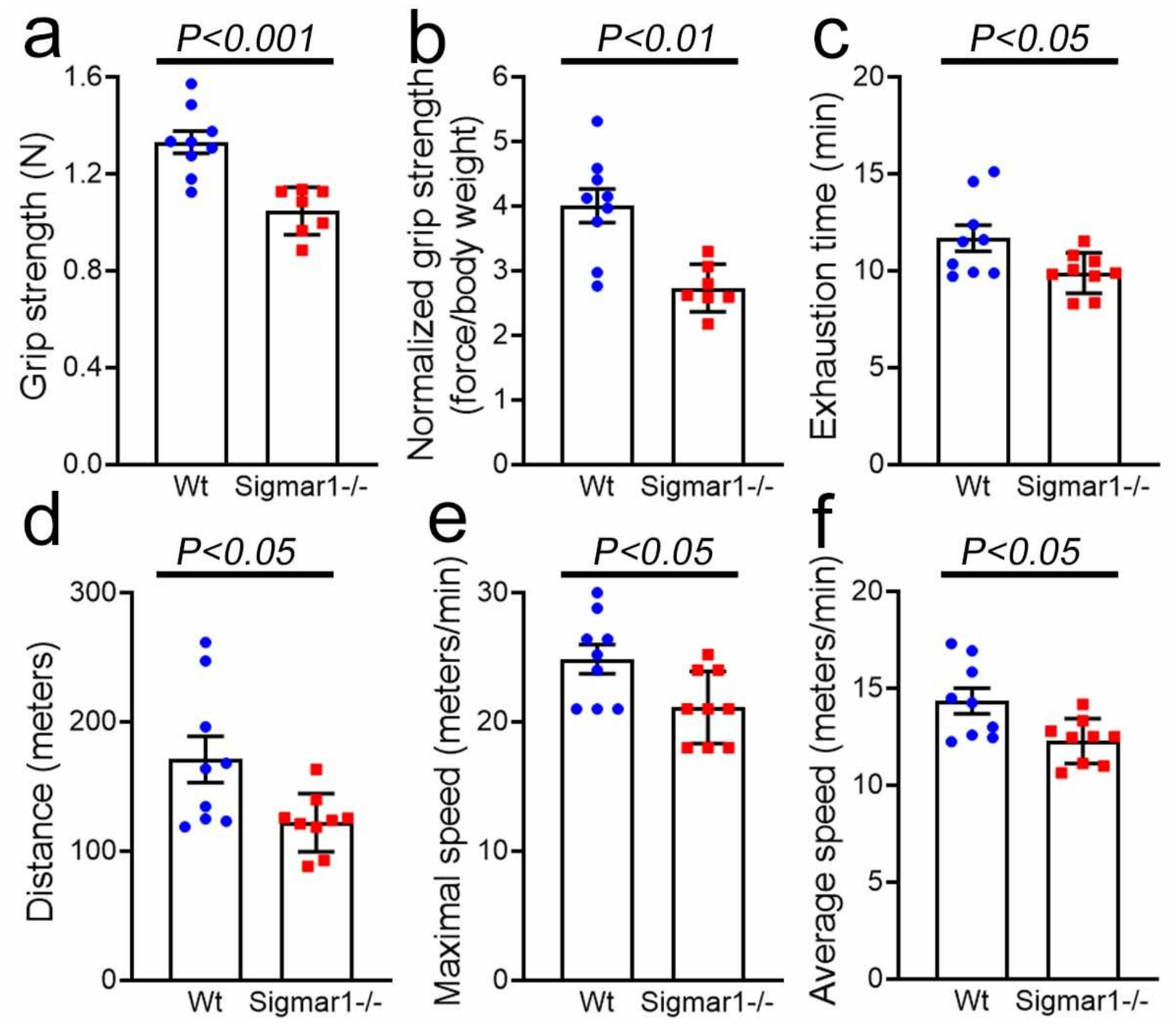

Figure 10

Sigmar1-/- mice showed reduced grip strength and exercise capacity compared to Wt mice. Wt and Sigmar1-/- mice were subjected to grip strength measurements and exercise tolerance tests to assess skeletal muscle function. Bar graphs represent summary data showing a absolute forelimb grip strength, $\mathrm{b}$ forelimb grip strength normalized to body weight, $\mathrm{c}$ time to exhaustion, $\mathrm{d}$ maximum running distance, $\mathrm{e}$ maximum speed attained, and $f$ average speed to assess endurance and exercise capacity of Sigmar1-/- 
mice. Measurement of the mice's grip strength and exercise capacity showed Sigmar1-/- mice have reduced endurance and reduced tolerance to exercise compared to Wt mice. Dots in the bar graphs represent individual values quantified for each $W t$ and Sigmar1-/- mice ( $n=7-9$ mice per genotype at 9-10 months of age). Data are expressed as mean \pm SEM. $P$ values were determined by unpaired Student's ttest. 